\title{
EXTENDING HOMEOMORPHISMS AND APPLICATIONS TO METRIC LINEAR SPACES WITHOUT COMPLETENESS
}

\author{
TADEUSZ DOBROWOLSKI
}

\begin{abstract}
A method of extending homeomorphisms between compacta metric spaces is presented. The main application is that homeomorphisms between compacta of an infinite-dimensional locally convex metric linear space extend to the whole space. A lemma used in the proof of this fact together with the method of absorbing sets is employed to show that every $\sigma$-compact normed linear space is homeomorphic to a dense linear subspace of a Hilbert space. A discussion of the relative topological equivalence of absorbing sets in noncomplete spaces is included. The paper is concluded with some controlled versions of an isotopy extension theorem.
\end{abstract}

\section{INTRODUCTION}

The first part of the paper is devoted to a question of whether homeomorphisms between two sets $K$ and $L$ of a space $X$ extend to the whole $X$. In the second part, we apply our results concerning this question to show the relative topological equivalence of certain $\sigma$-compact linear spaces in their completions. From this point of view, the most important case arises if $K$ and $L$ are compact and $X$ is a metric linear space. The first significant contribution in answering the question was a result of Klee [14]. It states that infinite-dimensional Hilbert spaces have the homeomorphism extension property for compacta, i.e., every homeomorphism between compact of an infinite-dimensional Hilbert space extends to the whole space. Since then the homeomorphism extension property has become central in investigations and applications of infinite-dimensional topology. Our objective is to establish the homeomorphism extension property for certain spaces $X$ without completeness.

The method we use goes back to the following "open action principle": if an action of a complete group on a complete space is almost open (i.e., closures of images of all open sets have nonempty interiors) then the action is open and transitive provided each of its orbits is dense. (Conversely, transitive actions

Received by the editors March 29, 1985 and, in revised form, February 19, 1988. Partially presented in the Special Session on Infinite Dimensional Topology at the annual meeting of the American Mathematical Society in Anaheim, California, January 1985.

1980 Mathematics Subject Classification (1985 Revision). Primary 57N17; Secondary 54C20, 46A05.

Key words and phrases. Homeomorphism extension property, almost extension property, locally convex linear space, skeleton sets. 
of separable metric groups are almost open on complete spaces.) The fact was discovered and communicated to the author by $\mathrm{H}$. Torunczyk (unpublished). It is an abstraction on the convergence procedure developed successively by Homma [13], Gluck [12], Bryant [5] and Stanko [16] which has its ancestor in the classical proof of the Banach open mapping principle due to Schauder. In our setting, we consider the natural action $c: H(X) \times E(K, X) \rightarrow E(K, X)$ of a group $H(X)$ of all homeomorphisms of $X$ on the space $E(K, X)$ of all embeddings of $K$ into $X$. To express the fact that $c$ is almost open and has each orbit dense we introduce the notion of the almost extension property. It requires, for every map $f: K \rightarrow X$ with $d(f$, id $)<\operatorname{dist}(K, X \backslash U)$, where $U$ is any neighborhood of $K$, and every $\varepsilon>0$, the existence of $h \in H(X)$ with $d(h \mid K, f)<\varepsilon, d(h, \mathrm{id})<d(f$, id $)+\varepsilon$ and $h \mid X \backslash U=\mathrm{id}$. Clearly, if $X$ is an infinite-dimensional linear space, $H(X)$ is not metrizable and the result on transitive actions is not directly applicable. However, under certain assumptions on $X$, a procedure of a proof of the above mentioned result can be adopted to show that $c$ is transitive. This yields the homeomorphism extension property of $X$. In the case of noncomplete $X$, one can consider its completion $\breve{X}$ and require additionally that $h$ of the almost extension property be a homeomorphism of $\check{X}$ preserving $X$. With this extra hypothesis we are able to extend a homeomorphism between $K$ and $L$ of $X$ to a homeomorphism of $\check{X}$ preserving $X$. Summarizing, a verification of the almost extension property is crucial in order to obtain the homeomorphism extension property. We have done this in the case of locally convex metric linear spaces $X$. The main result on extending homeomorphisms is that every homeomorphism between compacta $K$ and $L$ of an infinite-dimensional locally convex metric linear space $X$ extends to a homeomorphism $h$ of the completion $\check{X}$ such that $h$ preserves $X$. A smooth variation of this fact says that if additionally $X$ is a pre-Hilbert space, then the homeomorphism $h$ may be taken as a diffeomorphism of $\check{X} \backslash K$ onto $\check{X} \backslash L$.

In the application part of the paper we mostly deal with infinite-dimensional $\sigma$-compact linear spaces. We show that in such spaces, the homeomorphism extension property for the class of all $Z$-sets is true. Another application is an attempt to describe topological types of $\sigma$-compact linear spaces that are universal for a class of compacta. We prove that if locally convex metric linear spaces $E_{1}$ and $E_{2}$ are universal for a family of compacta $\mathscr{C}$ and $E_{1}$ and $E_{2}$ are countable unions of elements of $\mathscr{C}$, then they are homeomorphic. This generalizes the fact that maximal (resp. minimal) types of $\sigma$-compact linear spaces, i.e. universal for all compacta (resp., all finite-dimensional compacta), are homeomorphic, see [1, 3, 17 and 10]. As a corollary, we obtain the result that every infinite-dimensional locally convex metric linear space (resp., every infinite-dimensional normed linear space) which is $\sigma$-compact is homeomorphic to a dense linear subspace of the countable product of lines (resp., of a Hilbert space).

The next part of the paper deals with the problem of the relative topological equivalence of skeletoids in the sense of Bessaga-Pełczyński [3] (equivalently: 
absorbing sets in the sense of Anderson [1]) in noncomplete spaces $X$. In contrast to the case of complete $X$, we provide simple examples of homeomorphic skeletoids in standard $\sigma$-compact normed linear spaces $X$ which are not homeomorphic via a homeomorphism of the whole $X$. We have shown that, in certain instances, the theorem on the relative topological equivalence of skeletoids in noncomplete spaces holds true. For example, if a $\sigma$-compact locally convex metric linear space $E$ contains a Hilbert cube, then any $\sigma$-compact linear spaces $E_{1}$ and $E_{2}$ dense in $E$, which do not contain a Hilbert cube and are homeomorphic, are also homeomorphic via a homeomorphism of $E$, provided each $E \backslash E_{i}$ is $\sigma$-compact. (The spaces $E_{1}$ and $E_{2}$ are skeletoids for the class of all compacta which are embeddable in $E_{1}$ and $E_{2}$, respectively.) The basic method of constructing homeomorphisms in the application part is the standard method of skeletoids [3] (or absorbing sets [1]). Consequently, all homeomorphisms which we construct can be extended to the completions of spaces which we deal with.

In the last section of the paper we prove controlled versions of an isotopy extension theorem for infinite-dimensional locally convex metric linear spaces. It enables us to show that such spaces have the homeomorphism extension property for local compacta.

I am grateful to the referee for the suggestion to add a section on the controlled isotopy extension theorems. I also thank $\mathrm{H}$. Torunczyk for substantial discussions during the preparation of the final version of the paper.

\section{EXTENDING HOMEOMORPHISMS. AN ABSTRACT SCHEME}

Let $(X, d)$ be a metric space. Let us recall that $X$ is a neighborhood extensor for a class $\mathscr{A}$ of closed subsets of $X$ if

$\mathrm{NE}(\mathscr{A})$ : for every $A \in \mathscr{A}$ and every map $f: A \rightarrow X$, there exists a map $\bar{f}$ which is defined on a neighborhood of $A$ and which extends $f$.

Throughout this paper we will deal with a question of whether a homeomorphism $h: A \rightarrow B$ between subsets of $X$ extends to a homeomorphism of the whole $X$. Let $G$ be a subgroup of the group $H(X)$ of all homeomorphisms of $X$. The space $X$ is said to have the $G-\mathscr{A}$ extension property if every homeomorphism between subsets of $\mathscr{A}$ extends to a homeomorphism $h \in G$, see [4, p. 124]. It means that, for every $A \in \mathscr{A}$, the restriction map $h \rightarrow h \mid A$, $h \in G$, is onto. If the restriction map is an open map in the limitation topologies [20] on suitable function spaces, then $X$ is said to have the $G-\mathscr{A}$ estimated extension property, see [4]. We will be interested in the following version of the extension property for a class $\mathscr{K}$ of compacta of $X$. We say that $X$ has the $G-\mathscr{K}$ almost extension property if

$\operatorname{AEP}(G-\mathscr{K})$ : for every $K \in \mathscr{K}$, its neighborhood $U$ in $X$, a map $f: K \rightarrow X$ with $d(f$, id $)<\operatorname{dist}(K, X \backslash U)$ and every $\varepsilon>0$, there exists a homeomorphism $h \in G$ such that $d(h \mid K, f)<\varepsilon, d(h$, id $)<d(f$, id $)+\varepsilon$ and $h \mid X \backslash U=$ id . 
The above property is an estimated variation of the almost openness of the restriction map $h \rightarrow h \mid K$.

Given a subfamily $\mathscr{F}$ of the class $\mathscr{K}$, we say that $X$ has the $G-\mathscr{K}-\mathscr{F}$ limit flattening property if

$\operatorname{LFP}(G-\mathscr{K}-\mathscr{F})$ : for every $K \in \mathscr{K}$, its neighborhood $U$ in $X$ and every $\delta>0$, there exists a sequence $\left\{\phi_{n}\right\}$ of homeomorphisms of $X$ such that the uniform limit $\lim \phi_{n} \mid K=\phi_{\infty}$ is a map with $\phi_{\infty}(K) \in \mathscr{F}$ and, for every $n, d\left(\phi_{n}, \mathrm{id}\right)<\delta$ and $\phi_{n} \mid K \backslash U=\mathrm{id}$.

In infinite-dimensional topology the idea to "flatten" compact sets goes back to Klee [14], who observed that a compact subset of an infinite-dimensional Hilbert space can be considered as (a subset) of the graph of some map. Then the graph can be easily transformed via a homeomorphism of the whole space into a linear subspace of a nontrivial defect. This process is referred to as flattening a compact set. In our setting, the condition $\operatorname{LFP}(G-\mathscr{K}-\mathscr{F})$ guarantees flattening of each element of $\mathscr{K}$ to an element of $\mathscr{F}$.

Our main objective of this section is to establish the $G-\mathscr{K}$ extension property of a space $X$ with the $G-\mathscr{K}$ almost extension property. Consequently, the importance of detecting the $G-\mathscr{K}$ almost extension property arises. In many instances we find it easier to show $\operatorname{AEP}(G-\mathscr{F})$ for some subclass $\mathscr{F}$ of $\mathscr{K}$. It turns out that the $G-\mathscr{K}-\mathscr{F}$ limit flattening property reduces verifying $\operatorname{AEP}(G-\mathscr{K})$ to verifying $\operatorname{AEP}(G-\mathscr{F})$ together with $\operatorname{NE}(\mathscr{K})$. Our second goal of this section is to show this reduction.

We will start by noting the following remarks.

1.1. Remark. If $X$ satisfies $\operatorname{AEP}(G-\mathscr{K})$, then for every $K \in \mathscr{K}$, a map $f: K \rightarrow X$ can be arbitrarily closely approximating by restrictions $h \mid K, h \in G$. (We simply agree $\operatorname{dist}(K, \varnothing)=\infty$.)

1.2. Remark. The condition $\operatorname{AEP}(G-\mathscr{K})$ implies the following:

for every $K \in \mathscr{K}$, its neighborhood $U$ and a map $\varepsilon: X \rightarrow$ $(0, \infty)$, there exists $\alpha$ such that for every map $f: K \rightarrow X$ with $d(f$, id $)<\alpha$ can be arbitrarily closely approximated by restrictions $h \mid K$ with $h \in G, h \mid X \backslash U=$ id and $d(h(x), x)<\varepsilon(x)$.

The main result of this section reads as follows:

1.3. Theorem. Let $(X, d)$ be a metric space. Suppose $X$ has the $G-\mathscr{K}$ almost extension property for some class $\mathscr{K}$ of compacta of $X$ and some subgroup $G$ of $H(X)$. Then, every homeomorphism $h: A_{1} \rightarrow A_{2}$, where $A_{1}, A_{2} \in \mathscr{K}$, extends to a homeomorphism $\bar{h} \in G$. Moreover, $\bar{h}$ can be chosen so that, locally, $\bar{h} \mid X \backslash A_{1}$ and $(\bar{h})^{-1} \mid X \backslash A_{2}$ are finite compositions of elements of $G$.

Proof. We shall inductively find two sequences $\left\{h_{n}\right\}_{n=0}^{\infty}$ and $\left\{g_{n}\right\}_{n=0}^{\infty}$ of $G$ such that the sequences $\left\{\left(g_{n+1} \circ \cdots \circ g_{1} \circ g_{0}\right)^{-1} \circ h_{n} \circ \cdots \circ h_{1} \circ h_{0}\right\}$ and $\left\{\left(h_{n} \circ \cdots \circ h_{1} \circ h_{0}\right)^{-1} \circ g_{n+1} \circ \cdots \circ g_{1} \circ g_{0}\right\}$ are uniformly Cauchy with respect 
to the metric $d$ and stabilize outside $A_{1}$ and $A_{2}$, respectively. Moreover, we shall ensure that $\left\{\left(g_{n+1} \circ \cdots \circ g_{1} \circ g_{0}\right)^{-1} \circ\left(h_{n} \circ \cdots \circ h_{1} \circ h_{0}\right) \mid A_{1}\right\}$ converges to $h$ and $\left\{\left(h_{n} \circ \cdots \circ h_{1} \circ h_{0}\right)^{-1} \circ\left(g_{n+1} \circ \cdots \circ g_{1} \circ g_{0}\right) \mid A_{2}\right\}$ converges to $h^{-1}$. We shall finally set

$$
h(x)=\lim \left(g_{n+1} \circ \cdots \circ g_{1} \circ g_{0}\right)^{-1} \circ h_{n} \circ \cdots \circ h_{1} \circ h_{0}(x), \quad x \in X .
$$

\section{Write}

$$
\begin{gathered}
F_{k}^{1}=\left\{x \in X: \operatorname{dist}\left(x, A_{1}\right) \geq 2^{-k}\right\}, \quad F_{k}^{2}=\left\{x \in X: \operatorname{dist}\left(x, A_{2}\right) \geq 2^{-k}\right\}, \\
G_{k}^{1}=\left\{x \in X: \operatorname{dist}\left(x, h_{k} \circ \cdots \circ h_{1} \circ h_{0}\left(A_{1}\right)\right) \geq 2^{-k}\right\}
\end{gathered}
$$

and

$$
G_{k}^{2}=\left\{x \in X: \operatorname{dist}\left(x, g_{k} \circ \cdots \circ g_{1} \circ g_{0}\left(A_{2}\right)\right) \geq 2^{-k}\right\},
$$

for $k=1,2, \ldots$, and let $F_{0}^{1}=F_{0}^{2}=G_{0}^{1}=G_{0}^{2}=\varnothing$. To unify the inductive construction we fix the following notation. Let $\Phi_{n}=h_{k} \circ \cdots \circ h_{1} \circ h_{0}, F_{n}=F_{k}^{1}$ and $F_{n}^{\prime}=G_{k}^{1}$, for $n=2 k$, and let $\Phi_{n}=g_{k} \circ \cdots \circ g_{1} \circ g_{0}, F_{n}=F_{k}^{2}$ and $F_{n}^{\prime}=G_{k}^{2}$, for $n=2 k-1, k=0,1, \ldots$. We also write $u_{1}=h: A_{1} \rightarrow A_{2}$ and $u_{-1}=$ id: $A_{1} \rightarrow A_{1}$. With this notation we shall construct a sequence $\left\{\Phi_{n}\right\}_{n=-1}^{\infty}$ of $G$ to satisfy

$$
d\left(\Phi_{n-1}^{-1} \circ \Phi_{n} \circ u_{(-1)^{n-1}}, u_{(-1)^{n}}<2^{-n}\right.
$$

and

$$
d\left(\Phi_{n} \circ u_{(-1)^{n-1}}, \Phi_{n-1} \circ u_{(-1)^{n}}\right)<\alpha_{n},
$$

where $\alpha_{n}$ is that of $(*)$ (of 1.2) applied with

$$
K=\Phi_{n-1} \circ u_{(-1)^{n}}\left(A_{1}\right), \quad U=X \backslash\left(\Phi_{n-1}\left(F_{n-1}\right) \cup F_{n-1}^{\prime}\right)
$$

and

$$
\varepsilon=\varepsilon_{n-1}: X \rightarrow(0, \infty)
$$

such that $g \in H(X), d(g(x), x)<\varepsilon(x)$ imply

$$
\begin{aligned}
d^{*}\left(g \circ \Phi_{n-1}, \Phi_{n-1}\right)= & d\left(g \circ \Phi_{n-1}, \Phi_{n-1}\right) \\
& +d\left(\left(g \circ \Phi_{n-1}\right)^{-1}, \Phi_{n-1}^{-1}\right)<2^{-n}
\end{aligned}
$$

and

$$
d\left(\Phi_{n-2}^{-1} \circ \Phi_{n-1}, \Phi_{n-2}^{-1} \circ g \circ \Phi_{n-1}\right)<2^{-n},
$$

for $n=0,1,2, \ldots\left(\Phi_{-2}=\mathrm{id}\right)$.

$$
\Phi_{n}\left|F_{n-2} \cap \Phi_{n-2}^{-1}\left(F_{n-2}^{\prime}\right)=\Phi_{n-2}\right| F_{n-2} \cap \Phi_{n-2}^{-1}\left(F_{n-2}\right) \text {, }
$$

$$
\text { for } n=1,2, \ldots \text {, }
$$

$$
d^{*}\left(\Phi_{n}, \Phi_{n-2}\right)=d\left(\Phi_{n}, \Phi_{n-2}\right)+d\left(\Phi_{n}^{-1}, \Phi_{n-2}^{-1}\right)<2^{-n+1},
$$

$$
\text { for } n=1,2, \ldots \text {, }
$$




$$
d\left(\Phi_{n-3}^{-1} \circ \Phi_{n-2}, \Phi_{n-3}^{-1} \circ \Phi_{n}\right)<2^{-n+1}, \text { for } n=2,3, \ldots
$$

Inductive construction. Set $\Phi_{-1}=\mathrm{id}$ and let $n=0$. Pick $\alpha_{0}$ of the condition (*) applied to $K=\Phi_{-1} \circ u_{1}\left(A_{1}\right)=h\left(A_{1}\right), U=X$ and $\varepsilon_{-1}(x) \equiv \frac{1}{2}$. By Remark 1.1 , there exists $\Phi \in G$ such that $d\left(\Phi \mid A_{1}, h\right)<\alpha_{0}$. Letting $\Phi_{0}=\Phi$, we see that the condition (1) is satisfied for $n=0$. Suppose that $\Phi_{-1}, \Phi_{0}, \ldots, \Phi_{n-1}$ has been constructed for $n \geq 1$. Let $\alpha_{n}$ be that of $(*)$ applied with $\Phi_{n-1} \circ$ $u_{(-1)^{n}}\left(A_{1}\right), \quad U=X \backslash\left(\Phi_{n-1}\left(F_{n-1}\right) \cup F_{n-1}^{\prime}\right)$ and $\varepsilon=\varepsilon_{n-1}: X \rightarrow(0, \infty)$ such that $g \in H(X), d(g(x), x)<\varepsilon(x)$ imply $d^{*}\left(g \circ \Phi_{n-1}, \Phi_{n-1}\right)<2^{-n}$ and $d\left(\Phi_{n-2}^{-1} \circ \Phi_{n-1}, \Phi_{n-2}^{-1} \circ g \circ \Phi_{n-1}\right)<2^{-n}$. We assume that $\alpha_{n}$ is so small to fulfill the condition: $a \in \Phi_{n-1} \circ u_{(-1)^{n}}\left(A_{1}\right), d(x, a)<\alpha_{n}$ imply $d\left(\Phi_{n-1}^{-1}(x), \Phi_{n-1}^{-1}(a)\right)$ $<2^{-n}$. Writing

$$
\begin{aligned}
& K_{n-1}=\Phi_{n-2} \circ u_{(-1)^{n-1}}\left(A_{1}\right) \text { and } \\
& u_{n-1}=\Phi_{n-1} \circ u_{(-1)^{n-2}} \circ\left(\Phi_{n-2} \circ u_{(-1)^{n-1}}\right)^{-1} \mid K_{n-1},
\end{aligned}
$$

according to (1), we have $d\left(u_{n-1}\right.$, id $)<\alpha_{n-1}$. We also have $K_{n-1} \cap \Phi_{n-2}\left(F_{n-2}\right)$ $\cap F_{n-2}^{\prime}=\varnothing$. Thus by the property of $\alpha_{n-1}$, there exists a homeomorphism $g \in G$ such that

(a) $d\left(g \mid K_{n-1}, u_{n-1}\right)<\alpha_{n}$,

(b) $g \mid \Phi_{n-2}\left(F_{n-2}\right) \cup F_{n-2}^{\prime}=\mathrm{id}$,

(c) $d(g(x), x)<\varepsilon_{n-2}(x)$.

We set $\Phi_{n}=g \circ \Phi_{n-2}$. The condition (a) yields

$$
d\left(\Phi_{n} \circ u_{(-1)^{n-1}}, \Phi_{n-1} \circ u_{(-1)^{n}}\right)<\alpha_{n} ;
$$

hence, by the choice of $\alpha_{n}$,

$$
\begin{gathered}
d\left(\boldsymbol{\Phi}_{n-1}^{-1} \circ \boldsymbol{\Phi}_{n} \circ u_{(-1)^{n-1}}, \boldsymbol{\Phi}_{n-1}^{-1} \circ \boldsymbol{\Phi}_{n-1} \circ u_{(-1)^{n}}\right) \\
=d\left(\boldsymbol{\Phi}_{n-1}^{-1} \circ \boldsymbol{\Phi}_{n} \circ u_{(-1)^{n-1}}, u_{(-1)^{n}}\right)<2^{-n} .
\end{gathered}
$$

This, together with (b), shows that $\Phi_{n}$ satisfies (1) and (2). By the choice of $\varepsilon_{n-2}, \Phi_{n}$ also satisfies (3) and (4) (for $n \geq 2$ ). The inductive construction is complete.

We shall show that the sequences $\left\{\boldsymbol{\Phi}_{2 k+1}^{-1} \circ \boldsymbol{\Phi}_{2 k}(x)\right\}$ and $\left\{\boldsymbol{\Phi}_{2 k}^{-1} \circ \boldsymbol{\Phi}_{2 k+1}(x)\right\}$ converge $d$-uniformly in $X$ with respect to $x \in X$. Clearly then,

$$
\lim \left(\Phi_{2 k+1}^{-1} \circ \Phi_{2 k}\right) \text { and } \lim \left(\boldsymbol{\Phi}_{2 k}^{-1} \circ \Phi_{2 k+1}\right)
$$

are inverse maps of one another; and consequently $\bar{h}=\lim \left(\Phi_{2 k+1}^{-1} \circ \Phi_{2 k}\right)$ is a homeomorphism of $X$, see [4, p. 121]. Moreover, from the condition (1), we get $d\left(\Phi_{2 k}^{-1} \circ \Phi_{2 k+1} \circ h\right.$, id $)<2^{-2 k-1}$. This yields $\bar{h} \mid A_{1}=h$. A strategy to show that uniform limits of $\left\{\boldsymbol{\Phi}_{2 k+1}^{-1} \circ \boldsymbol{\Phi}_{2 k}\right\}$ and $\left\{\boldsymbol{\Phi}_{2 k}^{-1} \circ \boldsymbol{\Phi}_{2 k+1}\right\}$ are maps of $X$ into $X$ is to prove that the sequences: (i) are uniformly Cauchy, (ii) locally 
stabilize outside $A_{1}$ and $A_{2}$, and (iii) converge to $h$ and $h^{-1}$ on $A_{1}$ and $A_{2}$, respectively. Using (3) and (4), we estimate

$$
\begin{aligned}
d\left(\boldsymbol{\Phi}_{2 k+1}^{-1} \circ \boldsymbol{\Phi}_{2 k}, \boldsymbol{\Phi}_{2 k+3}^{-1} \circ \boldsymbol{\Phi}_{2 k+2}\right) \leq d\left(\boldsymbol{\Phi}_{2 k+1}^{-1} \circ \boldsymbol{\Phi}_{2 k}, \boldsymbol{\Phi}_{2 k-1}^{-1} \circ \boldsymbol{\Phi}_{2 k}\right) \\
\quad+d\left(\boldsymbol{\Phi}_{2 k-1}^{-1} \circ \boldsymbol{\Phi}_{2 k}, \boldsymbol{\Phi}_{2 k-1}^{-1} \circ \boldsymbol{\Phi}_{2 k+2}\right)+d\left(\boldsymbol{\Phi}_{2 k-1}^{-1} \circ \boldsymbol{\Phi}_{2 k+2}, \boldsymbol{\Phi}_{2 k+3}^{-1} \circ \boldsymbol{\Phi}_{2 k+2}\right) \\
=d\left(\boldsymbol{\Phi}_{2 k+1}^{-1}, \boldsymbol{\Phi}_{2 k-1}^{-1}\right)+d\left(\boldsymbol{\Phi}_{2 k-1}^{-1} \circ \boldsymbol{\Phi}_{2 k}, \boldsymbol{\Phi}_{2 k-1}^{-1} \circ \boldsymbol{\Phi}_{2 k+2}\right)+d\left(\boldsymbol{\Phi}_{2 k-1}^{-1}, \boldsymbol{\Phi}_{2 k+3}^{-1}\right) \\
\leq 2^{-2 k}+2^{-2 k-1}+d\left(\boldsymbol{\Phi}_{2 k-1}^{-1}, \boldsymbol{\Phi}_{2 k+1}^{-1}\right)+d\left(\boldsymbol{\Phi}_{2 k+1}^{-1}, \boldsymbol{\Phi}_{2 k+3}^{-1}\right) \\
<2^{-2 k}+2^{-2 k-1}+2^{-2 k}+2^{-2 k-2}<5 \cdot 2^{-2 k} .
\end{aligned}
$$

This shows that $\left\{\Phi_{2 k+1}^{-1} \circ \Phi_{2 k}(x)\right\}$ is a Cauchy sequence uniformly with respect to $x \in X$. To conclude that $\left\{\Phi_{2 k}^{-1} \circ \Phi_{2 k+1}(x)\right\}$ is a Cauchy sequence we similarly observe, via (3) and (4), that

$$
\begin{aligned}
d\left(\boldsymbol{\Phi}_{2 k}^{-1} \circ \boldsymbol{\Phi}_{2 k+1}, \boldsymbol{\Phi}_{2 k+2}^{-1} \circ \boldsymbol{\Phi}_{2 k+3}\right) \\
\quad \leq d\left(\boldsymbol{\Phi}_{2 k}^{-1} \circ \boldsymbol{\Phi}_{2 k+1}, \boldsymbol{\Phi}_{2 k}^{-1} \circ \boldsymbol{\Phi}_{2 k+3}\right)+d\left(\boldsymbol{\Phi}_{2 k}^{-1} \circ \boldsymbol{\Phi}_{2 k+3}, \boldsymbol{\Phi}_{2 k+2}^{-1} \circ \boldsymbol{\Phi}_{2 k+3}\right) \\
\quad \leq 2^{-2 k-2}+d\left(\boldsymbol{\Phi}_{2 k}^{-1}, \boldsymbol{\Phi}_{2 k+2}^{-1}\right) \leq 2^{-2 k-2}+2^{-2 k-1}<2^{-2 k} .
\end{aligned}
$$

It was already noticed that $\left\{\Phi_{2 k+1}^{-1} \circ \Phi_{2 k} \mid A_{2}\right\}$ converges uniformly to $h^{-1}$. We have

$$
\begin{aligned}
d\left(\boldsymbol{\Phi}_{2 k+1}^{-1} \circ \boldsymbol{\Phi}_{2 k} \mid A_{1}, h\right) \leq & d\left(\boldsymbol{\Phi}_{2 k+1}^{-1} \circ \boldsymbol{\Phi}_{2 k}, \boldsymbol{\Phi}_{2 k+3}^{-1} \circ \boldsymbol{\Phi}_{2 k+2}\right) \\
& +d\left(\boldsymbol{\Phi}_{2 k+3}^{-1} \circ \boldsymbol{\Phi}_{2 k+2}, \boldsymbol{\Phi}_{2 k+1}^{-1} \circ \boldsymbol{\Phi}_{2 k+2}\right) \\
& +d\left(\boldsymbol{\Phi}_{2 k+1}^{-1} \circ \boldsymbol{\Phi}_{2 k+2} \mid A_{1}, h\right) .
\end{aligned}
$$

The above estimate together with (1) yields the convergence of $\left\{\boldsymbol{\Phi}_{2 k+1}^{-1} \circ \Phi_{2 k} \mid A_{1}\right\}$ to $h$.

Thus, it remains to show that $\left\{\Phi_{2 k+1}^{-1} \circ \Phi_{2 k}\right\}$ and $\left\{\Phi_{2 k}^{-1} \circ \Phi_{2 k+1}\right\}$ locally stabilize off $A_{1}$ and $A_{2}$, respectively. Pick $x_{0} \notin A_{1}$. By (2), there exists a neighborhood $U_{0}$ of $x_{0}$ with $U_{0} \cap A_{1}=\varnothing$, and $n_{0}$ such that $h_{n} \circ \cdots \circ h_{n_{0}} \circ$ $\cdots \circ h_{1} \circ h_{0}\left|U_{0}=h_{n_{0}} \circ \cdots \circ h_{1} \circ h_{0}\right| U_{0}$, we claim that there exists a neighborhood $V_{0} \subset h_{n_{0}} \circ \cdots \circ h_{1} \circ h_{0}\left(U_{0}\right)$ of $y_{0}=h_{n_{0}} \circ \cdots \circ h_{1} \circ h_{0}\left(x_{0}\right)$ such that $V_{0} \subset G_{k}^{2}$ for almost all $k$. Otherwise, there would exist a sequence $\left\{y_{k_{l}}\right\} \subset h_{n_{0}} \circ \cdots \circ h_{1} \circ h_{0}\left(U_{0}\right)$ convergent to $y_{0}$ and such that $d\left(y_{k_{l}}, g_{k_{l}} \circ \cdots \circ g_{1} \circ g_{0}\left(a_{k_{l}}\right)\right)<2^{-k_{l}}$ for some $a_{k_{l}} \in A_{2}$. By the compactness of $A_{2}$ we would assume that $\left\{a_{k_{l}}\right\}$ converges to some $a \in A_{2}$. Consequently, the sequence $\left\{g_{k_{l}} \circ \cdots \circ g_{1} \circ g_{0}\left(a_{k_{l}}\right)\right\}$ converges to $y_{0}$. Therefore for $k_{l}$ large, we have

$$
\begin{aligned}
& \left(h_{k_{l}-1} \circ \cdots \circ h_{1} \circ h_{0}\right)^{-1} \circ\left(g_{k_{l}} \circ \cdots \circ g_{1} \circ g_{0}\right)\left(a_{k_{l}}\right) \\
& \quad=\left(h_{n_{0}} \circ \cdots \circ h_{1} \circ h_{0}\right)^{-1} \circ\left(g_{k_{l}} \circ \cdots \circ g_{1} \circ g_{0}\right)\left(a_{k_{l}}\right) ;
\end{aligned}
$$

the latter sequence convergent to $x_{0}$. On the other hand

$$
\left\{\left(h_{k_{l}-1} \circ \cdots \circ h_{1} \circ h_{0}\right)^{-1} \circ\left(g_{k_{l}} \circ \cdots \circ g_{1} \circ g_{0}\right)\left(a_{k_{l}}\right\}\right.
$$


converges to $h^{-1}(a)$, contradicting the fact that $x_{0} \notin A_{2}$. Thus, for $k \geq k_{0}$, we have $V_{0} \subset h_{n_{0}} \circ \cdots \circ h_{1} \circ h_{0}\left(U_{0}\right) \cap G_{k}^{2}$ and, by virtue of $(2), g_{k} \mid V_{0}=$ id for $k \geq k_{0}$. Hence, for every $y \in V_{0}$ and $k \geq k_{0}+1$, we have $g_{k} \circ \cdots \circ g_{k_{0}+1} \circ g_{k_{0}}(y)=y$; so letting $z=\left(g_{k_{0}-1} \circ \cdots \circ g_{0}\right)^{-1}(y)$, we get $g_{k} \circ \cdots \circ g_{1} \circ g_{0}(z)=y$. Thus, for $y=h_{k_{0}} \circ \cdots \circ h_{1} \circ h_{0}(x) \in V_{0}, x \in U_{0}$, we obtain

$$
\begin{aligned}
z & =\left(g_{k_{0}+1} \circ \cdots \circ g_{1} \circ g_{0}\right)^{-1} h_{k_{0}} \circ \cdots \circ h_{1} \circ h_{0}(x) \\
& =\left(g_{k+1} \circ \cdots \circ g_{1} \circ g_{0}\right)^{-1} \circ h_{k} \circ \cdots \circ h_{1} \circ h_{0}(x)
\end{aligned}
$$

provided $k_{0} \geq n_{0}$. The same argument shows that $\left\{\left(h_{k} \circ \cdots \circ h_{1} \circ h_{0}\right)^{-1}\right.$ $\left.\circ g_{k+1} \circ \cdots \circ g_{1} \circ g_{0}\right\}$ locally stabilizes outside $A_{2}$. From this it follows that, locally $\bar{h}$ and $(\bar{h})^{-1}$ are finite compositions of elements of $G$ on $X \backslash A_{1}$ and $X \backslash A_{2}$, respectively. The proof is complete.

In the case where $(X, d)$ is a complete metric space the above proof can be simplified. In this case the uniform limits of $\left\{h_{n} \circ \cdots \circ h_{1} \circ h_{0}\right\}$ and $\left\{g_{n} \circ\right.$ $\left.\cdots \circ g_{1} \circ g_{0}\right\}$ will be homeomorphisms of $X$ and $\lim g_{n} \circ \cdots \circ g_{1} \circ g_{0}(h(a))=$ $\lim h_{n} \circ \cdots \circ h_{1} \circ h_{0}(a)$ for $a \in A_{1}$. Hence $\left(\lim g_{n} \circ \cdots \circ g_{1} \circ g_{0}\right)^{-1} \circ \lim h_{n} \circ \cdots \circ$ $h_{1} \circ h_{0}$ is a required homeomorphism $\bar{h}$. To show that $\lim \left(h_{n} \circ \cdots \circ h_{1} \circ h_{0}\right)$ and $\lim \left(g_{n} \circ \cdots \circ g_{1} \circ g_{0}\right)$ are homeomorphisms we do not need the stabilizing condition (2). Hence the extension $\bar{h} \in H(X)$ exists even if the stabilizing condition of 1.2 is dropped. However to construct homeomorphisms $h \in H(X)$ with $d(h(x), x)<\varepsilon(x)$ for arbitrary $\varepsilon: X \rightarrow(0, \infty)$ and noncompact $X$ we usually require $h$ to be the identity off some open subset of $X$.

1.4. Note. Suppose $X_{0}$ is a subspace of $X$ such that $g\left(X_{0}\right)=X_{0}$ for every $g \in G$ (i.e., $X_{0}$ is $G$-invariant). Then, in 1.3 we additionally obtain that the pairs $\left(X_{0}, X_{0} \backslash A_{1}\right)$ and $\left(\left(X_{0} \backslash A_{2}\right) \cup h\left(A_{1} \cap X_{0}\right), X_{0} \backslash A_{2}\right)$ are homeomorphic. Moreover, by the last part of $1.3, \bar{h} \mid X_{0} \backslash A_{1}$ and $(\bar{h})^{-1} \mid X_{0} \backslash A_{2}$ are locally finite compositions of $g \mid X_{0}$ with $g \in G$. Consequently, if $A_{1}, A_{2} \subset X_{0}$ then $\bar{h} \mid X_{0}$ is a homeomorphism of $X_{0}$ extending $h$.

1.5. Theorem. Let $(X, d)$ be a metric space, $G$ be a subgroup of $H(X), \mathscr{K}$ a class of compacta of $X$ and $\mathscr{F}$ its subclass. Assume $X$ has the $G-\mathscr{K}-\mathscr{F}$ limit flattening property and is a neighborhood extensor for $\mathscr{K}$. If $X$ has the $G-\mathscr{F}$ almost extension property, then it has the $G-\mathscr{K}$ almost extension property.

Proof. Let $K \in \mathscr{K}$, its neighborhood $U$ and $\varepsilon>0$ be given. Consider a map $f: K \rightarrow X$ such that $d(f$, id $)<d_{0}=\operatorname{dist}(K, X \backslash U)$. We may assume that $f$ extends to $\bar{f}$ over $U$. There exists $\delta>0$ with $\delta<\varepsilon / 4$ and such that for $k \in K, d(x, k) \leq \delta$ implies $d(\bar{f}(x), f(k))<\varepsilon / 4$. With this $\delta$, pick a sequence $\left\{\varphi_{n}\right\}$ given by the condition $\operatorname{LFP}(G-\mathscr{K}-\mathscr{F})$. Assume 
$\varepsilon<d_{0}-d\left(f\right.$, id) and write $f^{\prime}=\bar{f} \mid F$, where $F=\varphi_{\infty}(K) \in \mathscr{F}$. We have

$$
\begin{aligned}
d\left(f^{\prime}, \mathrm{id}\right) & \leq d\left(\bar{f} \varphi_{\infty}, f\right)+d(f, \mathrm{id})+d\left(\mathrm{id}, \varphi_{\infty}\right) \\
& \leq \varepsilon / 4+d(f, \mathrm{id})+\varepsilon / 4<d_{0}-\varepsilon / 4 \\
& <\operatorname{dist}(F, X \backslash U) .
\end{aligned}
$$

Now, the condition $\operatorname{AEP}(G-\mathscr{F})$ is applicable with $\left(F, U, f^{\prime}\right)$. So, there exists $h^{\prime} \in G, h^{\prime} \mid X \backslash U=\mathrm{id}$ and $d\left(h^{\prime}\right.$, id $)<d\left(f^{\prime}\right.$, id $)+\varepsilon / 4$. We shall find a required homeomorphism $h$ of $\operatorname{AEP}(G-\mathscr{K})$ in the form $h=h_{n}=h^{\prime} \circ \varphi_{n}$. First of all observe that

$$
\begin{aligned}
d\left(h_{n}, \mathrm{id}\right) & =d\left(h^{\prime} \varphi_{n}, \mathrm{id}\right) \leq d\left(h^{\prime} \varphi_{n}, \varphi_{n}\right)+d\left(\varphi_{n}, \mathrm{id}\right) \\
& <d\left(h^{\prime}, \mathrm{id}\right)+\varepsilon / 4<d\left(f^{\prime}, \mathrm{id}\right)+\varepsilon / 2 \\
& \leq d(f, \mathrm{id})+\varepsilon / 2+\varepsilon / 2=d(f, \mathrm{id})+\varepsilon .
\end{aligned}
$$

Next, by the uniform convergence of $\left\{\varphi_{n} \mid K\right\}$ there exists $n_{0}$ such that for every $n \geq n_{0}, d\left(h^{\prime} \varphi_{n} \mid K, h^{\prime} \varphi_{\infty}\right)<\varepsilon / 4$. This enables us to estimate for $n \geq n_{0}$

$$
\begin{aligned}
d\left(h_{n} \mid K, f\right) & =d\left(h^{\prime} \varphi_{n} \mid K, f\right) \\
& \leq d\left(h^{\prime} \varphi_{n} \mid K, h^{\prime} \varphi_{\infty}\right)+d\left(h^{\prime} \mid K, f^{\prime}\right)+d\left(\bar{f} \varphi_{\infty}, f\right) \\
& \leq \varepsilon / 4+\varepsilon / 4+\varepsilon / 4<\varepsilon .
\end{aligned}
$$

Since both $h^{\prime}$ and $\varphi_{n_{0}}$ are the identity outside $U, h_{n_{0}}$ also is. We complete the proof, letting $h=h_{n_{0}}$.

1.6. Note. An inspection of the proof of 1.6 yields the following observation: Assume $K \in \mathscr{K}$ and its neighborhood $U$ satisfy $\operatorname{LFP}(G-\mathscr{K}-\mathscr{F})$, and suppose every map $K$ into $X$ admits a neighborhood extension. Then, if for every $F \in \mathscr{F}$ with $F \subset U$, mapping $f^{\prime}: F \rightarrow U$, and $\varepsilon>0$ one can find $h^{\prime} \in G$ with $d\left(h^{\prime} \mid F, f^{\prime}\right)<\varepsilon, h^{\prime} \mid X \backslash U=\mathrm{id}$, then for every $f: K \rightarrow U$ and $\varepsilon>0$ one can find $h \in G$ with $d(h \mid K, f)<\varepsilon, h \mid X \backslash U=$ id and $d(h$, id $)<d(f$, id $)+\varepsilon$.

\section{Applications-The topological CASE}

In our applications of the results 1.3 and $1.5 X$ will be a metric linear space (briefly: m.l.s.). In the case where $X$ is a locally convex metric linear space (briefly: 1.c.m.l.s.) we are able to show the homeomorphism extension property for compacta. We have done very little in the case where the local convexity is dropped. In the applications, $\mathscr{K}$ will be the class of all compacta of $X$ and $\mathscr{F}$ will be the class of all compacta contained in finite-dimensional linear subspaces of $X$. We also use the class $\mathscr{F} d$ of all finite-dimensional compacta of $X$. Note that $\mathscr{F} \subset \mathscr{F} \subset \mathscr{K}$. We always consider $X$ with a metric $d$ induced by an $F$-norm; hence, $d$ is translation invariant, and $d(t x, 0) \leq d(x, 0)$ for every $x \in X$ and $|t| \leq 1$. If $X_{0}$ is a dense linear subspace of $X$, then by $H\left(X \mid X_{0}\right)$ we denote the subgroup of $H(X)$ of homeomorphisms preserving every linear subspace of $X$ containing $X_{0}$. 
We start with

2.1. Lemma. Let $X$ be an infinite-dimensional m.l.s. and $X_{0}$ its dense linear subspace. Then, $X$ has the $G-\mathscr{F}$ almost extension property, where $G=$ $H\left(X \mid X_{0}\right)$ and $\mathscr{F}$ is the class of all compacta contained in linear finite-dimensional subspaces of $X_{0}$.

Proof. Let $F \in \mathscr{F}$, its neighborhood $U$ and $f: F \rightarrow X$ with $d(f$, id $)<d_{0}=$ $\operatorname{dist}(F, X \backslash U)$ be given. First, we easily show that $f$ can be arbitrarily closely approximated by embeddings $u: F \rightarrow X_{0}$ with $u(F) \in \mathscr{F}$. Approximate $f$ by $f^{\prime}$ such that $\operatorname{span}\left(\operatorname{im} f^{\prime}\right)=X^{\prime} \subset X_{0}$. Then, since $X_{0}$ is infinite-dimensional, there is a finite-dimensional linear subspace $X^{\prime \prime}$ of $X_{0}$ admitting embeddings $v: F \rightarrow X^{\prime \prime}$ with $d(v, 0)$ as small as we wish and such that $X^{\prime \prime} \cap X^{\prime}=\{0\}$. It is clear that $u$ can be taken as $f^{\prime}+v$.

Consider a finite-dimensional linear subspace $X_{1}$ of $X_{0}$ with $X^{\prime}+X^{\prime \prime} \subset X_{1}$ and $\operatorname{dim}\left(X_{1}\right) \geq 2 \operatorname{dim}\left(X^{\prime}+X^{\prime \prime}\right)+3$. If $u$ is close to $f$, then $d(u$, id $)<$ $\operatorname{dist}(F, X \backslash U)$ and $u_{t}(x)=(1-t) x+t u(x), 0 \leq t \leq 1$, joins $\mathrm{id}_{F}$ with $u$ in $X_{1} \cap U$. The space $X_{1}$ has the estimated homeomorphism extension property for compacta $A \subset X_{1}$ with $\operatorname{dim}(\operatorname{span} A) \leq \operatorname{dim}\left(X^{\prime}+X^{\prime \prime}\right)+1$. Therefore, given $\varepsilon>0$ there is an isotopy $\left(\Phi_{t}\right)$ of $X_{1}$ such that $\Phi_{0}=\mathrm{id}, d\left(\Phi_{1} \mid F, u\right)<\varepsilon$, $d\left(\Phi_{t}\right.$, id $)<d(u$, id $)+\varepsilon$ and $\Phi_{t} \mid X \backslash U=$ id for all $0 \leq t \leq 1$.

We shall extend $\Phi_{1}$ to a homeomorphism of $X$ preserving every linear subspace containing $X_{0}$. By a result of Michael [15], there exists a continuous right inverse $\alpha: X / X_{1} \rightarrow X$ for the quotient map $\kappa: X \rightarrow X / X_{1}$ such that $\alpha(0)=0$. Moreover, the formula $h_{0}(x)=(\kappa(x), x-\alpha \kappa(x))$ defines a homeomorphism of $X$ onto $\left(X / X_{1}\right) \times X_{1}$ transforming $X_{1}$ identically onto $\{0\} \times X_{1}$. Also, we have $h_{0}(Y)=\left(Y / X_{1}\right) \times X_{1}$ for every linear subspace $Y$ of $X$ with $Y \supset X_{1}$. Consider neighborhoods $V$ of the origin in $X / X_{1}$ and $U^{\prime}$ of $\operatorname{im}\left(u_{t}\right)$ in $X_{1}$ such that $V \times U^{\prime} \subset h_{0}(U)$. Pick a map $\lambda: X / X_{1} \rightarrow[0,1]$ such that $\lambda(0)=1$ and $\lambda(y)=0$ for $y \notin V$. For $(y, x) \in\left(X / X_{1}\right) \times X_{1}$, write $\Phi(y, x)=\left(y, \Phi_{\lambda(y)}(x)\right)$. Clearly, $\Phi$ is a homeomorphism extending $h_{0} u h_{0}^{-1}$ and $\Phi$ is the identity outside $h_{0}(U)$. Consequently, $h=h_{0}^{-1} \Phi h_{0}$ extends $u$ and is the identity outside $U$. We complete the proof by noting that $d(h, \mathrm{id})<d(u$, id $)+\varepsilon$ and that $h \in H\left(X \mid X_{0}\right)$.

2.2. Note. Under the assumptions of 2.1 one can fulfill the condition $\operatorname{AEP}(G-\mathscr{K})$ with the requirement $d(f$, id $)<\operatorname{dist}(K, X \backslash U)$ dropped, provided $U$ is the complement of a compact subset $L$ of $X$. This follows from the fact that $L$ is a $Z$-set in $X$. Consequently, there exists a homotopy $\left(f_{t}\right): F \rightarrow U=X \backslash K$ with $f_{0}=$ id and $f_{1}$ as close to $f$ as we wish. Next, we approximate $\left(f_{t}\right)$ by embeddings $u: F \times[0,1] \rightarrow X \backslash K$ with $u_{0}=$ id and $\operatorname{im}(u) \in \mathscr{F}$. Now we may follow the argument of the proof of 2.1 .

2.3. Lemma. Let $E$ be an infinite-dimensional l.c.m.l.s. and $E_{0}$ its dense linear subspace. Then, $E$ has the $H\left(E \mid E_{0}\right)-\mathscr{K}-\mathscr{F}$ limit flattening property, 
where $\mathscr{K}$ is the class of all compacta of $E$ and $\mathscr{F}$ is the class of all compacta contained in finite-dimensional linear subspaces of $E_{0}$.

Proof. Fix $K \in \mathscr{K}$, its neighborhood $U$ and $\delta>0$. We may assume that $\delta<\operatorname{dist}(K, E \backslash U)$. Pick a finite-dimensional linear subspace $E_{1}$ of $E_{0}$ with $K \subset E_{1}+\{x \in E: d(x, 0)<\delta / 4\}$. Consider a continuous right inverse $\alpha$ for the quotient map $\kappa: E \rightarrow E / E_{1}$ [15]. By the local convexity we may additionally require $d(\alpha(y), 0) \leq 2 \bar{d}(y, 0)$ for all $y \in E / E_{1}$, where $\bar{d}$ is the quotient metric of $d$. Write $h_{0}(x)=(\kappa(x), x-\alpha \kappa(x))$ for $x \in E$. For every $k \in K$ and every $x \in E$ with $d(\kappa(x), 0) \leq d(\kappa(k), 0)$, we have $(\kappa(x), k-\alpha \kappa(k)) \in h_{0}(U)$. Otherwise $h_{0}^{-1}(\kappa(x), k-\alpha \kappa(k)) \in E \backslash U$ and we would get

$$
\begin{aligned}
d(\alpha \kappa(x)+k-\alpha \kappa(k), k) & =d(\alpha \kappa(x)-\alpha \kappa(k), 0) \\
& \leq 4 \bar{d}(\kappa(k), 0)<\delta<\operatorname{dist}(K, E \backslash U),
\end{aligned}
$$

a contradiction. The above shows that, using a partition of unity argument, we can construct a map $\omega: E_{1} \rightarrow[0, \delta / 4]$ with

$$
h_{0}(K) \subset\left\{(y, x) \in\left(E / E_{1}\right) \times E_{1}: \bar{d}(y, 0)<\omega(x)\right\} \subset h_{0}(U) .
$$

Let $\alpha_{t}:[0, \infty] \rightarrow(0,1], 0 \leq t<1$, be a homotopy with $\alpha_{0} \equiv 1, \alpha_{t}$ monotone, $\alpha_{t} \mid[1, \infty] \equiv 1$ for each $t$, and for every $s<1, \lim _{t \rightarrow 1} \alpha_{t}(s)=0$. Define an isotopy $\left(g_{t}\right)$ of $\left(E / E_{1}\right) \times E_{1}, 0 \leq t<1$, by letting $g_{t}(y, x)=$ $\left(\alpha_{t}(\bar{d}(y, 0) / \omega(x)) y, x\right)$ for $y \neq 0$ and $g_{t}(0, x)=(0, x)$. Observe that for each $0 \leq t<1, \Lambda_{t}=h_{0}^{-1} g_{t} h_{0} \in H\left(E \mid E_{0}\right), d\left(\Lambda_{t}, \mathrm{id}\right)<\delta, \Lambda_{t} \mid E \backslash U=\mathrm{id}$, and

$$
\lim _{t \rightarrow 1} \Lambda_{t}(k)=k-\alpha \kappa(k) \in E_{1}
$$

uniformly with respect to $k \in K$. We set $\varphi_{n}=\Lambda_{1-(1 / n)}$ to fulfill $\operatorname{LFP}(G-\mathscr{K}-\mathscr{F})$.

Now, we shall prove our main result on extending homeomorphisms.

2.4. Theorem. Let $E$ be an infinite-dimensional l.c.m.l.s. and $\check{E}$ its completion. Every homeomorphism $h: K \rightarrow L$ between compacta of $E$ extends to a homeomorphism $h \in H(\check{E} \mid E)$.

Proof. Let $\mathscr{K}$ be the class of all compacta and $\mathscr{F}$ the class of all compacta contained in finite-dimensional subspaces of $E$. The space $\check{E}$ satisfies

$$
\operatorname{AEP}(H(E \mid E)-\mathscr{F}) \text { and } \operatorname{LFP}(H(E \mid E)-\mathscr{K}-\mathscr{F})
$$

by 2.1 and 2.3 , respectively. The local convexity of $\check{E}$ implies that $\check{E}$ is an AR (see [4, p. 67]) and consequently it satisfies $\mathrm{NE}(\mathscr{K})$. Hence, Theorem 1.5 is applicable and $\check{E}$ satisfies $\operatorname{AEP}(H(\check{E} \mid E)-\mathscr{K})$. Now, the assertion of theorem follows from 1.3 and 1.4.

For the purpose of the future citation let us note the following facts. 
2.5. Proposition. Let $E$ be an infinite-dimensional l.c.m.l.s. and $E_{0}$ its dense linear subspace. For every compact set $K \subset E$, its neighborhood $U$ and a map $\varepsilon: E \rightarrow(0, \infty)$, there exists a $\delta>0$ such that every map $f: K \rightarrow E$ with $d(f$, id $)<\delta$ can be arbitrarily closely approximated by restrictions $h \mid K$, where $h \in H\left(E \mid E_{0}\right)$ satisfies $d(h(x), x)<\varepsilon(x)$ and $h \mid E \backslash U=\mathrm{id}$.

Proof. An argument of the proof of Theorem 2.4 shows that $E$ satisfies $\operatorname{AEP}\left(H\left(E \mid E_{0}\right)-\mathscr{K}\right)$. This yields the assertion via Remark 1.2.

2.6. Proposition (cf. [10]). Let $E$ be an infinite-dimensional l.c.m.l.s. and $E_{0}$ its dense linear subspace. For every disjoint pair of compacta $K$ and $L$ of $E$, mapping $f: K \rightarrow E$, and $\varepsilon>0$, there exists a homeomorphism $h \in H\left(E \mid E_{0}\right)$ with $d(f, h \mid K)<\varepsilon, d(h, \mathrm{id})<d(f$, id $)+\varepsilon$ and $h \mid L=\mathrm{id}$.

Proof. Follow the proof of 2.5. The needed changes are those of 2.2 and 1.6.

We do not know whether Lemma 2.3 remains true without the local convexity. Observe that the condition $\operatorname{LFP}(\mathscr{K}-\mathscr{F})$ (or more generally: $\operatorname{LFP}(\mathscr{K}-\mathscr{F} d)$ ) implies $\operatorname{NE}(\mathscr{K})$ for any m.l.s. $X$. For, if $X$ satisfies $\operatorname{LFP}(\mathscr{K}-\mathscr{F} d)$, then for every $K \in \mathscr{K}, \mathrm{id}_{K}$ is the uniform limit of a sequence $\phi_{n}: K \rightarrow X$ with $\phi_{n}(K) \in \mathscr{F}$; consequently, by a result of [8], $X$ is an absolute extensor for compacta. Since, in spite of few attempts, the problem of whether all m.l.s. $X$ are extensors for compacta is still unsettled, we suspect that also verifying $\operatorname{LFP}(\mathscr{K}-\mathscr{F} d)$ is not simple. It is reasonable to ask

2.7. Question. Assume an infinite-dimensional m.l.s. $X$ is an absolute extensor for the class of all compacta $\mathscr{K}$. Does $X$ satisfy $\operatorname{LFP}(\mathscr{K}-\mathscr{F} d)$ ?

Summarizing, not only do we not know if all m.l.s. $X$ have the homeomorphism extension property for compacta, but we do not know this for the class $\mathscr{F} d$ of all finite-dimensional compacta either. It is not clear how to verify the condition $\operatorname{LFP}(\mathscr{F} d-\mathscr{F})$ for arbitrary $X$. In the case where $X$ is complete, the homeomorphism extension property for compacta implies that $X$ is an absolute extensor for compacta. Below we show that the complete $X$ has the homeomorphism extension property for the class $\mathscr{F} d$.

2.8. Theorem. Every infinite-dimensional complete m.l.s. $X$ satisfies the following version of the $\mathscr{F} d-H(X)$ estimated homeomorphism extension property: for every $K \in \mathscr{F}$, its neighborhood $U$ and an embedding $v: K \rightarrow X$ with $d(v$, id $)<\operatorname{dist}(K, X \backslash U)$ and $\varepsilon>0$, there exists $h \in H(X)$ extending $v$, and satisfying $d(h, \mathrm{id})<d(v, \mathrm{id})+\varepsilon$ and $h \mid X \backslash U=\mathrm{id}$.

We shall need:

2.9. Lemma. Every infinite-dimensional complete m.l.s. $X$ has the homeomorphism extension property for finite-dimensional compacta.

Sketch of proof. Let $K, L \in \mathscr{F} d$ and let $h: K \rightarrow L$ be a homeomorphism. Since $\operatorname{span}(K \cup L)$ is $\sigma$-compact and $X$ is complete, there exists a finitedimensional linear subspace $E$ of $X$ with $\operatorname{dim}(E) \geq 2 \operatorname{dim}(K)+1$ and such 
that $E \cap \operatorname{span}(K \cup L)=\{0\}$. Consider the homeomorphism

$$
h_{0}(x)=(\kappa(x), x-\alpha \kappa(x))
$$

of $X$ onto $(X / E) \times E$, where $\kappa: X \rightarrow X / E$ is the quotient map and $\alpha: X / E \rightarrow X$ its cross section (we use a Michael result of [15]). Clearly, $h_{0}(K \cup L)$ is the graph of a map of a finite-dimensional compact subset of $X / E$ into $E$. Now, the result follows in a standard way by using Klee's method [14] (see also [7]). (Note that, under our assumptions on the dimension of $E, L$ can be embedded in $E$.)

Proof of 2.8. We shall employ a method of Anderson and McCharen of [2]. First, observe that the homotopy $u_{t}=(1-t)$ id $+t v, 0 \leq t \leq 1$, joins id $_{K}$ with $v$ in $U$ with $d\left(u_{t}\right.$, id $) \leq d\left(v\right.$,id). Since $\operatorname{span}\left(\operatorname{im}\left(u_{t}\right)\right)$ is $\sigma$-compact we can approximate $\left(u_{t}\right)$ by $\left(v_{t}\right)$ such that $v_{0}=\mathrm{id}, v_{1}=v$ and $(k, t) \rightarrow$ $\left(v_{t}(k), t\right)$ is an embedding. Pick a finite-dimensional linear subspace of $X$ with $\operatorname{dim}(E) \geq 2 \operatorname{dim}(K)+1$ and a vector $x \in X \backslash E$. Let $Y=X / E \oplus \mathbf{R} x$ and $h_{0}$ be a homeomorphism of $X$ onto $Y \times E \oplus \mathbf{R} x=Y \times E \times \mathbf{R}$ given in the same fashion as in the proof of 2.6. If $i: K \rightarrow E$ is any embedding, write $e(k, t)=(i(k), t)$ for an embedding of $K \times[0,1]$ into $\{0\} \times E \times R \subset Y \times E \times R$. By virtue of 2.9, there eixsts a homeomorphism $\bar{h}: X \rightarrow E \times \mathbf{R} \times Y$ such that $\bar{h} v(k, t)=e(k, t),(k, t) \in K \times[0,1]$. Given $\varepsilon>0$, let

$$
U(k)=\bigcup_{0 \leq t \leq 1}\{x \in U: d(x, v(k, t))<\varepsilon / 2\} .
$$

Clearly, we have $e\left(\{k\} \times[0,1] \subset \bar{h}^{-1}(U(k))\right.$ for every $k \in K$. Using the $\mathbf{R}$-coordinate only, we construct a homeomorphism $\Phi$ of $Y \times E \times \mathbf{R}$ with $\Phi(y, e(k, 0))=(y, e(k, 1)),(y, k) \in Y \times K$ and such that $\Phi=$ id outside $\bigcup_{k \in K} \bar{h}(U(k))$. Moreover, we can require that for each $x \in \bigcup_{k} U(k)=U^{\prime}$ there corresponds $k_{0} \in K$ with $\bar{h}(x) \in \bar{h}\left(U\left(k_{0}\right)\right)$ and $\Phi(\bar{h}(x)) \in \bar{h}\left(U\left(k_{0}\right)\right)$. We set $h=\bar{h}^{-1} \Phi \bar{h}$. Since $h(x)=x$ for every $x \notin U^{\prime}, h \mid X \backslash U=$ id. Furthermore, if $x \in U^{\prime}$ then for some $k_{0} \in K$ we have $\bar{h}^{-1} \Phi \bar{h}(x), x \in U\left(k_{0}\right)$; consequently $d(x, h(x))<\operatorname{diam}\left(U\left(k_{0}\right)\right) \leq \varepsilon+d\left(v\left(k_{0}, t\right), v\left(k_{0}, s\right)\right)$. Thus, if $\left(v_{t}\right)$ is close enough to $\left(u_{t}\right)$, we get $d(h$, id $)<\varepsilon+d(v$, id $)$. To finish the proof observe that $h \mid K=v$.

2.10. Remark. Every infinite-dimensional complete m.l.s. $X$ has the $\mathscr{F} d-H(X)$ almost extension property. This can be easily derived from 2.8 , because by the $\sigma$-compactness of $f(K)$, every map $f: K \rightarrow X, K \in \mathscr{F} d$, can be arbitrarily closely approximated by embeddings.

Note that an affirmative answer to 2.7 , together with $2.10,1.5$, and 1.3 will imply the extension homeomorphism property for compact of $X$.

We close this section by proving that every infinite-dimensional l.c.m.l.s. $E$ has the $\mathscr{K}-H(\check{E} \mid E)$ estimated homeomorphism extension property (in the version of [4]). Note that to prove this, we cannot apply the Anderson-McCharen 
technique [2] because we do not know whether compact sets are deficient in possibly noncomplete $E$. Instead, we shall make use of Toruńczyk's result [17]. A set $K \subset E \subset \check{E}$ is said to be $H(\check{E} \mid E)$-thin [4] if, for every neighborhood $U$, the identity $\mathrm{id}_{E}$ can be arbitrarily closely approximated by $h \in H(\check{E} \mid E)$ with $h \mid \check{E} \backslash U=$ id and $h(K) \cap K=\varnothing$.

2.11. Lemma. Every compact subset $K$ of an infinite-dimensional l.c.m.l.s. $E$ is $H(\check{E} \mid E)$-thin.

Proof. Since the compact subsets of every infinite-dimensional m.l.s. are $Z$ sets, $\mathrm{id}_{K}$ can be approximated by maps $K \rightarrow(E \cap U) \backslash K$. Now, the result follows from the $\mathscr{K}-H(\check{E} \mid E)$ almost extension property of $\check{E}$ (see 2.4).

A result of [17] together with 2.11 yields

2.12. Theorem. Every infinite-dimensional l.c.m.l.s. $E$ has the $\mathscr{K}-H(\check{E} \mid E)$ estimated homeomorphism extension property.

2.13. Note. The version of the estimated homeomorphism property formulated in 2.8 also holds true in the above case. We could prove it without referring to Toruńczyk's theorem. Observe that once we choose $h_{0}$ in the proof of 1.3 we determine the inductive process in the sense that $h_{1}, h_{2}, \ldots$ and $g_{0}, g_{1}, \ldots$ can be chosen as close to the identity as we please.

\section{Applications-The SMOOTH CASE}

In this section we shall be interested in the question of whether a homeomorphism $h: K \rightarrow L$ between compacta of a normed linear space $E$ (briefly: n.l.s.) extends to a homeomorphism $\bar{h}$ of $E$ such that $\bar{h}: E \backslash K \rightarrow E \backslash L$ is a diffeomorphism. The technique of Theorem 1.3 enables us to discuss this question without referring to the Inverse Function Theorem. Consequently, it allows us to drop the completeness assumption on $E$ in [7] and to prove the first result in this area without completeness.

We start with a lemma whose proof is a smooth analogue of that of 2.8 .

3.1. Lemma. Let $E$ be an infinite-dimensional n.l.s. and $E_{0}$ be its dense linear subspace. If $E$ admits a $C^{p}$ norm, then $E$ has the $\operatorname{Diff}^{p}\left(E \mid E_{0}\right)-\mathscr{F}$ almost extension property, where $\mathscr{F}$ is a class of all compacta contained in finite-dimensional linear subspaces of $E_{0}$ and $\operatorname{Diff}^{p}\left(E \mid E_{0}\right)$ is the group of all $C^{p}$ diffeomorphisms of $E$ preserving every linear space containing $E_{0}$.

Proof. We shall make the necessary changes in the proof of 2.8 that are required by the smooth case. Let $K \in \mathscr{F}$, its neighborhood $U$ and a map $f: K \rightarrow$ $E$ with $d(f$, id $)<\operatorname{dist}(K, E \backslash U)=d_{0}$ be given. Since $\operatorname{span}(K)$ is finitedimensional there exists a $C^{\infty}$ manifold $M$ with $K \subset M$ and $K \cap \partial M=$ $\varnothing$ such that $f$ admits an extension $\bar{f}: M \rightarrow E_{0}$ with $d(\bar{f}$, id $)<d_{0}$ and $\bar{f}(M) \in \mathscr{F}$. We may additionally require that $M \subset U$. Consider a homotopy $f(m, t)=(1-t) m+\bar{t} \bar{f}(m)$, for $m \in M$ and $-\delta<t<1+\delta$, with $\delta>0$ chosen so that $\operatorname{im} f \subset U$. Include $M \cup \bar{f}(M)$ in a finite-dimensional linear subspace 
$E_{1}$ of large dimension. If $3\left(\operatorname{dim} E_{1}+1\right)>\operatorname{dim}(M)$, then $f: M \times(-\delta, 1+\delta) \rightarrow$ $U \cap E_{1}$ can oe closely approximated by $C^{\infty}$ embeddings $g: M \times(-\delta, 1+\delta) \rightarrow$ $U \cap E_{1}$ with $g \mid M \times\{0\}=$ id. Hence, we may assume that $\bar{f}=f(\cdot, 1)$ and $g(\cdot, 1)$, and $d\left(g_{t}\right.$, id $)$ and $d\left(f_{t}\right.$, id $)$ are as close as we wish, respectively. Split $E_{1}=Y \oplus E^{\prime} \oplus \mathbf{R}$ in such a way that $M$ admits a $C^{\infty}$ embedding $i: M \rightarrow E^{\prime}$. Write $e(m, t)=(0, i(m), t)$ for a $C^{\infty}$ embedding of $M \times(-\delta, 1+\delta)$ into $Y \oplus E \oplus \mathbf{R}$. We may assume that $\operatorname{dim}\left(E_{1}\right)$ is so large that embeddings $g$ and $e$ are $C^{\infty}$ diffeotopic in $E_{1}$. Then, by the classic theorem of Thom there exists a $C^{\infty}$ diffeomorphism $h^{\prime}: E_{2} \rightarrow Y \times E^{\prime} \oplus \mathbf{R}$ such that $h^{\prime} g=e$. Consider any splitting $E=E^{\prime} \oplus E_{1}$ and write $h_{0}\left(x^{\prime}+x_{1}\right)=x^{\prime}+h\left(x_{1}\right)$ for $x^{\prime}+x_{1} \in E^{\prime} \oplus E_{1}$. Next, as in the proof of 2.8, construct a homeomorphism $\Phi$ of $E^{\prime} \oplus Y \oplus E \oplus \mathbf{R}$ with $\Phi(y, m, 0)=(y, m, 1), d\left(h_{0}^{-1} \Phi h_{0}(x), x\right)<d\left(g_{t}\right.$, id $)+\varepsilon$ and $h_{0}^{-1} \Phi h_{0} \mid E \backslash U=$ id. Since $E$ admits a $C^{p}$ norm, we can easily perform this construction to obtain $\Phi$ as a $C^{p}$ diffeomorphism. The proof is completed because $h_{0}^{-1} \Phi h_{0} \mid M=g(\cdot, 1)$ is close to $\bar{f}$ and $\bar{f} \mid K=f$.

3.2. Lemma. Let $H$ be an infinite-dimensional pre-Hilbert space and $H_{0}$ its linear dense subspace. Then $H$ has the $\operatorname{Diff}^{\infty}\left(H \mid H_{0}\right)-\mathscr{K}-\mathscr{F}$ limit flattening property, where $\mathscr{K}$ is the class of all compacta of $H$ and $\mathscr{F}$ is the class of all compacta contained in finite-dimensional linear subspaces of $H_{0}$.

Proof. We shall follow the proof of 2.3. Let $K \in \mathscr{K}$, its neighborhood $U$ and $\delta>0$ be given. Assume $\delta<\operatorname{dist}(K, H \backslash U)$. There is an orthogonal splitting $H=H_{1} \oplus H^{\prime}$ such that a finite-dimensional space $H_{1}$ is contained in $H_{0}$ and $x_{1}+x_{2} \in K, x_{1} \in H_{1}, x_{2} \in H^{\prime}$ imply $\left\|x_{2}\right\|<\delta / 2$. Using the fact that Hilbert spaces admit smooth partitions of unity [18], we construct a $C^{\infty}$ map $\omega: H_{1} \rightarrow[0, \delta / 2]$ with

$$
K \subset\left\{x_{1}+x_{2} \in H_{1} \oplus H^{\prime}:\left\|x_{2}\right\|<\omega\left(x_{1}\right)\right\} \subset U .
$$

Consider the homotopy $\left(\alpha_{t}\right)$ of the proof of 2.3. We additionally may require that each $\alpha_{t}$ is $C^{\infty}$ smooth and $\alpha_{t} \equiv 0$ on a neighborhood of 0 . Write $g_{t}\left(x_{1}+x_{2}\right)=x_{1}+\alpha_{t}\left(\left\|x_{2}\right\| / \omega\left(x_{1}\right)\right) x_{2}$ for $x_{2} \neq 0$ and $g_{t}\left(x_{1}\right)=x_{1}$. Since each $g_{t}, 0 \leq t<1$, is a $C^{\infty}$ diffeomorphism, we set $\varphi_{n}=g_{1-(1 / n)}$ to fulfill $\operatorname{LFP}\left(\operatorname{Diff}^{\infty}\left(H \mid E_{0}\right)-\mathscr{K}-\mathscr{F}\right)$.

3.3. Lemma. Let $E$ be a Banach space with a Schauder basis. Assume $E$ admits a $C^{p}$ norm. Then $E$ has the $\operatorname{Diff}^{p}(E)-\mathscr{K}-\mathscr{F}$ limit flattening property.

Proof. Let $K \in \mathscr{K}$, its neighborhood $U$ and $\delta>0$ be given. Let $\left\{\left(e_{i}, x_{i}^{*}\right)\right\}_{i=1}^{\infty}$ be a Schauder basis of $E$. With $\delta<\operatorname{dist}(K, E \backslash U)$, there exists $n$ such that $\left\|\sum_{k \geq n} x_{k}^{*}(x) e_{k}\right\|<\delta / 2$ for every $x \in K$. Let $P_{1}(x)=\sum_{k=1}^{n} x_{k}^{*}(x) e_{k}$ and $P_{2}(x)=x-P_{1}(x)$. Replacing the splitting $H=H_{1} \oplus H^{\prime}$ by $E=P_{1}(E) \oplus P_{2}(E)$ we may now proceed as in the proof of 3.2 . 
Our main result on smooth extension of homeomorphism is

3.4. Theorem. Let $H$ be an infinite-dimensional pre-Hilbert space and $\breve{H}$ its completion. Every homeomorphism $h: K \rightarrow L$ between compacta of $H$ can be extended to $\bar{h} \in H(\breve{H} \mid H)$ such that $\bar{h}$ is a $C^{\infty}$ diffeomorphism on $\check{H} \backslash K$.

Proof. This follows from 3.1, 3.2 and 1.5 applied to 1.3 (see also 1.4).

As a consequence we obtain the following results on $C^{\infty}$ extracting of compact sets from pre-Hilbert spaces.

3.5. Corollary. Every compact subset $K$ of an infinite-dimensional pre-Hilbert space $H$ is $C^{\infty}$ negligible in $H$, i.e., there exists a $C^{\infty}$ diffeomorphism $h: H \backslash K \underset{\text { onto }}{\longrightarrow} H$.

Proof. The result is that of [7] if $H$ is complete. So, with $\check{H}$ to be the completion of $H$, we may assume $x \in \check{H} \backslash H$. Consider the map $k \rightarrow x+k$ of $K$ into $\breve{H} \backslash H$. Using 3.4, this map can be extended to a homeomorphism $\bar{h}$ of $\breve{H}$ such that $\bar{h} \mid \breve{H} \backslash K$ is a $C^{\infty}$ diffeomorphism. Note that $\bar{h}$ sends $H \backslash K$ onto $H \backslash(x+K)=H$ (see 1.4).

Employing 3.3 instead of 3.2 in the proof of 3.4 we obtain

3.6. Theorem. Let $E$ be a Banach space with a Schauder basis. Assume $E$ admits a $C^{p}$ norm. Then, every homeomorphism $h: K \rightarrow L$ between compacta of $E$ extends to a homeomorphism $\bar{h}$ of $E$ such that $\bar{h}$ is a $C^{p}$ diffeomorphism of $E \backslash K$ onto $E \backslash L$.

\section{Minimal TYPES OF M.L.S. THAT ARE UNIVERSAL FOR A CLASS OF COMPACTA ARE HOMEOMORPHIC}

Every infinite-dimensional m.l.s. which is a countable union of finite-dimensional compacta is universal for the class of finite-dimensional compacta. Every such space is homeomorphic to $l_{2}^{f}$, a linear subspace of $l_{2}$ consisting of all finite sequences; see [3]. Also every $\sigma$-compact 1.c.m.l.s. which is universal for the class of all compacta is homeomorphic to $\Sigma$, the linear span of the Hilbert cube $Q=\left\{\left(x_{i}\right) \in l_{2}:\left|x_{i}\right| \leq 1 / i\right\}$ in $l_{2}$; see [10]. The following fact generalizes these two results.

4.1. Theorem. Let $E_{1}$ and $E_{2}$ be l.c.m.l.s. universal for a class of compacta $\mathscr{T}$. The spaces $E_{1}$ and $E_{2}$ are homeomorphic iff $E_{1}, E_{2} \in \mathscr{T}_{\sigma}$, where $\mathscr{T}_{\sigma}$ is the class of sets which are countable unions of elements of $\mathscr{T}$.

We shall prove a slightly general fact.

4.2. Proposition. Expressing $E_{i}=\bigcup_{1}^{\infty} A_{n}^{i}$ as unions of compacta, assume each $A_{n}^{1}$ can be embedded in $E_{2}$ and each $A_{n}^{2}$ can be embedded in $E_{1}$. Then, there exists a homeomorphism $h: \check{E}_{1} \rightarrow \check{E}_{2}$ of the completions of $E_{i}$ 's with $h\left(E_{1}\right)=E_{2}$.

The proof will employ the skeleton technique of [1, 3 and 17]. We shall need the following lemma which is a refinement of a result of [10]. 
4.3. Lemma. Let $(A, B)$ be a pair of compacta such that $A$ admits an embedding in a l.c.m.l.s. $E$. Given a compact set $K \subset E, \varepsilon>0$ and a map $f:(A, B) \rightarrow(\check{E}, E)$ such that $f \mid B$ is an embedding, there exists an embedding $v: A \rightarrow E$ with $v|B=f| B, d(v, f)<\varepsilon$ and $v(A \backslash B) \cap K=\varnothing$.

$\operatorname{Proof}$ (cf. [10]). Since $\check{E} \backslash E$ is locally homotopy negligible in $\check{E}, f: A \rightarrow \check{E}$ can be arbitrarily closely approximated by maps equal to $f$ on $B$ whose ranges are in $E$. Therefore, we shall assume $f(A) \subset E$. We also shall assume that $A \subset E$, possibly with $A \cap(K \cup f(A))=\varnothing$. Represent $A \backslash B=\bigcup_{0}^{\infty} A_{i}$, where $\varnothing=A_{0} \subset A_{1} \subset A_{2} \subset \cdots$ is a tower of compacta. Given $\varepsilon>0$, we shall construct a sequence $\left\{h_{n}\right\}_{n=0}^{\infty} \subset H(E)$ such that for $n \geq 1$ we have

(1) $d\left(h_{n}, h_{n-1}\right)<2^{-n+1} \varepsilon$;

(2) $h_{n}\left|A_{n-1}=h_{n-1}\right| A_{n-1}$ and $h_{n} \mid f(A) \cup K=$ id ;

(3) $d\left(h_{n}|B, f| B\right)<2^{-n-1} \varepsilon$.

To construct $h_{0}$ apply 2.6 with $K \equiv A$ and $L=f(A) \cup K$. If $h_{0}, h_{1}, \ldots, h_{n-1}$ $(n \geq 1)$ are already constructed, by applying 2.6 with $f h_{n-1}^{-1} \mid h_{n-1}(B), L=$ $h_{n-1}\left(A_{n-1}\right) \cup f(A) \cup K$, we obtain a homeomorphism $h$. Clearly, $h_{n}=h \circ h_{n-1}$ satisfies $(1)-(3)$.

Set $v(a)=\lim h_{n}(a), a \in A$. Conditions (1)-(3) imply that $v$ is a continuous one-to-one map of $A$ into $E$ with $v|B=f| B$. By (2) and the fact that $A \cap(K \cap f(A))=\varnothing$, we infer that $v(A \backslash B) \cap K=\varnothing$.

Proof of 4.2. By the Kadec-Anderson-Torunczyk Theorem [20], the completions $\check{E}_{1}$ and $\check{E}_{2}$ are homeomorphic. Let $d_{1}$ and $d_{2}$ be metrics of $\check{E}_{1}$ and $\check{E}_{2}$, respectively. The method of skeletoids [3] requires us to construct sequences of compacta $\left\{C_{n}^{1}\right\}_{n=1}^{\infty}$ of $E_{1}$ and $\left\{C_{n}^{2}\right\}_{n=1}^{\infty}$ of $E_{2}$ and a sequence of homeomorphisms $h_{n}: \check{E}_{1} \rightarrow \check{E}_{2}, n=1,2, \ldots$, such that

(1) $A_{n}^{1} \subset C_{n}^{1}$ and $A_{n}^{2} \subset h_{n}^{1}\left(C_{n}^{1}\right) \subset C_{n}^{2}$,

(2) $h_{n}\left|C_{n-1}^{1}=h_{n-1}\right| C_{n-1}^{1}$;

(3) $d^{*}\left(h_{n}, h_{n-1}\right)=d_{2}\left(h_{n}, h_{n-1}\right)+d_{1}\left(h_{n}^{-1}, h_{n-1}^{-1}\right) \leq 2^{-n}(n \geq 2)$.

Clearly, the condition (3) implies that $h=\lim h_{n}$ and $g=\lim h_{n}^{-1}$ exist and $g$ and $h$ are inverse maps of one another. Hence, $h$ is a homeomorphism of $\check{E}_{1}$ onto $\check{E}_{2}$ sending $E_{1}$ onto $E_{2}$ (use (2) and (3)).

The inductive construction. With $A_{1}^{1}=C_{1}^{1}=A_{1}^{2}=C_{1}^{2}=\varnothing$, let $h_{1}$ be any homeomorphism of $\check{E}_{1}$ onto $\check{E}_{2}$. Assume $h_{1}, h_{2}, \ldots, h_{n-1}(n \geq 2)$ have been constructed. Apply Lemma 4.3 with $A=A_{n}^{1}, B=C_{n-1}^{1} \cap A_{n}^{1}, K=h_{n-1}\left(C_{n-1}^{1}\right)$ and $f=h_{n-1} \mid A_{n}^{1}$, to approximate $f$ by an embedding $v: A_{n}^{1} \rightarrow E_{2}$ satisfying $v|B=f| B$ and $v\left(A_{n}^{1} \backslash C_{n-1}^{1}\right) \cap h_{n-1}\left(C_{n-1}^{1}\right)=\varnothing$. Thus, by letting $v\left|C_{n-1}^{1}=h_{n-1}\right| C_{n-1}^{1}, v$ extends to an embedding over the whole $C_{n-1}^{1}$. Applying the estimated homeomorphism extension property [4, p. 153] (see also 2.5), we find a homeomorphism $h: \check{E}_{1} \rightarrow \check{E}_{2}$ such that $h\left|C_{n-1}^{1}=h_{n-1}^{1}\right| C_{n-1}^{1}$, $h\left(C_{n-1}^{1} \cup A_{n}^{1}\right)=v\left(C_{n-1}^{1} \cup A_{n}^{1}\right) \subset E_{2}$ and $d^{*}\left(h, h_{n}\right)<2^{-n-1}$. Next apply 
Lemma 4.3 with $A=A_{n}^{2}, \quad B=A_{n}^{2} \cap h\left(C_{n-1}^{1} \cup A_{n}^{2}\right), \quad K=h\left(C_{n-1}^{1} \cup A_{n}^{2}\right)$ and $f=h^{-1} \mid A_{n}^{2}$, to approximate $f$ by an embedding $v: A_{n}^{2} \rightarrow E_{2}$ with $v\left|B=h^{-1}\right| B$ and $v\left(A_{n}^{2} \backslash h\left(C_{n-1}^{1} \cup A_{n}^{1}\right)\right) \cap\left(C_{n-1}^{1} \cup A_{n}^{1}\right)=\varnothing$. Again by letting $v\left|h\left(C_{n-1}^{1} \cup A_{n}^{1}\right)=h^{-1}\right| h\left(C_{n-1}^{1} \cup A_{n}^{1}\right), v$ extends to an embedding over $h\left(C_{n-1}^{1} \cup A_{n}^{1}\right)$. By using the estimated homeomorphism extension property we extend $v$ to a homeomorphism $g: \check{E}_{2} \rightarrow \check{E}_{1}$ such that $d^{*}\left(g, h^{-1}\right)<2^{-n-1}$. Finally, we set $C_{n}^{1}=C_{n-1}^{1} \cup A_{n}^{1} \cup g\left(A_{n}^{2}\right), C_{n}^{2}=h\left(C_{n-1}^{1} \cup A_{n}^{1}\right) \cup A_{n}^{2}$ and $h_{n}=g^{-1}$. Conditions (1)-(3) are fulfilled.

The following fact is a direct consequence of 4.2.

4.4. Corollary. Let $E_{1}$ and $E_{2}$ be infinite-dimensional $\sigma$-compact l.c.m.l.s. and $\check{E}_{1}, \check{E}_{2}$ their completions. If there exists a one-to-one map of $E_{1}$ onto $E_{2}$, then the pairs $\left(\check{E}_{1}, E_{1}\right)$ and $\left(\check{E}_{2}, E_{2}\right)$ are homeomorphic.

4.5. Corollary. Every infinite-dimensional $\sigma$-compact l.c.m.l.s. [resp. n.l.s.] $E$ is homeomorphic to a dense linear subspace of the countable product of lines $\mathbf{R}^{N}$ [resp. of the Hilbert space $l_{2}$ ]. In either case there exists a homeomorphism which can be extended to the completions.

Proof. Let $\left\{x_{n}^{*}\right\}_{n=1}^{\infty}$ be a sequence of continuous linear functionals separating the points of $E$. Consider $T(x)=\left(x_{i}^{*}(x)\right), x \in E$. Clearly, $T$ is a continuous one-to-one map of $E$ into $\mathbf{R}^{N}$. By $4.5, E$ is homeomorphic to $T(E)$. In the case where $E$ is a normed space, we may require additionally that $\left\|x_{n}^{*}\right\| \leq$ $1 / n, n=1,2, \ldots$, so that $T$ will map $E$ into $l_{2}$. Now, the first assertion of 4.5 follows from the fact that every closed infinite-dimensional linear subspace of $\mathbf{R}^{N}$ [resp. of $l_{2}$ ] is isomorphic to $\mathbf{R}^{N}$ [resp. to $l_{2}$ ] itself. The second assertion is a consequence of 4.4 .

Below, we apply the skeleton technique to show the homeomorphism extension property for $Z$-sets in every l.c.m.l.s. which is $\sigma$-compact.

4.6. Theorem. Every homeomorphism $h: F_{1} \rightarrow F_{2}$ between $Z$-sets of an infinitedimensional $\sigma$-compact l.c.m.l.s. $E$ extends to a homeomorphism $\bar{h}$ of $E$.

Proof. Let $\check{E}$ be the completion of $E$. It follows from the Lavrientieff theorem that $h$ extends to $\tilde{h}: \widetilde{F}_{1} \rightarrow \widetilde{F}_{2}$, where $\widetilde{F}_{i}$ is a $G_{\delta}$ subset of $\operatorname{cl}\left(F_{i}\right) \subset \check{E}, i=1,2$. There is a $G_{\delta}$ subset $\widetilde{E}$ of $\check{E}$ with $E \subset \widetilde{E}$, and $\widetilde{F}_{1}$ and $\widetilde{F}_{2}$ are closed in $\widetilde{E}$. (Let $\rho$ be a complete metric on $\widetilde{F}_{1} \cup \widetilde{F}_{2}$. Since $\widetilde{F}_{1} \cup \widetilde{F}_{2}$ is closed in $\widetilde{F}_{1} \cup \widetilde{F}_{2} \cup E$, by a Hausdorff theorem [4, p. 62] $\rho$ extends to $\widetilde{F}_{1} \cup \widetilde{F}_{2} \cup E$. The completion $\left(\widetilde{F}_{1} \cup \widetilde{F}_{2} \cup E, \rho\right)$ may serve as a suitable $\widetilde{E}$.) The space $\widetilde{E}$ as a complement of a $\sigma Z$-set in $\check{E}$ is homeomorphic to $\mathbf{R}^{N}$ [4, p. 166]. Clearly, $\widetilde{F}_{1}$ and $\widetilde{F}_{2}$ are $Z$-sets in $\widetilde{E}$. Therefore, by a theorem of Anderson [4, p. 165] $\tilde{h}$ can be extended to a homeomorphism $h_{0}$ of $\widetilde{E}$.

We shall modify $h_{0}$ in order to get $h_{0}(E)=E$. Represent $E \backslash F_{1}=\bigcup_{1}^{\infty} A_{n}^{1}$ and $E \backslash F_{2}=\bigcup_{1}^{\infty} A_{n}^{2}$, where $A_{n}^{i}$ are compacta. We shall inductively construct 
sequences of compacta $\left\{C_{n}^{1}\right\}_{n=1}^{\infty}$ and $\left\{C_{n}^{2}\right\}_{n=1}^{\infty}$ and a sequence of homeomorphisms $\left\{h_{n}\right\}_{n=1}^{\infty}$ of $\widetilde{E}$ such that for $n \geq 1$ we have

(1) $A_{n}^{1} \subset C_{n}^{1} \subset E \backslash F_{1}, A_{n}^{2} \subset h_{n}\left(C_{n}^{1}\right) \subset C_{n}^{2} \subset E \backslash F_{2}$;

(2) $h_{n}\left|C_{n-1}^{1}=h_{n-1}\right| C_{n-1}^{1}$ and $h_{n}\left|\widetilde{F}_{1}=h_{0}\right| \widetilde{F}_{1}$;

(3) $d^{*}\left(h_{n}, h_{n-1}\right)=d\left(h_{n}, h_{n-1}\right)+d\left(h_{n}^{-1}, h_{n-1}^{-1}\right) \leq 2^{-n}$, where $d$ is a complete metric on $\widetilde{E}$.

Finally, we set $\bar{h}=\lim h_{n} \mid E$.

With $A_{1}^{i}=C_{1}^{i}=\varnothing(i=1,2)$, we let $h_{1}=h_{0}$. Suppose $h_{1}, \ldots, h_{n-1}(n \geq 2)$ have been constructed. We make use of 4.3 to approximate $h_{n-1} \mid C_{n-1}^{1} \cup A_{n}^{1}$ by embeddings $v: C_{n-1}^{1} \cup A_{n}^{1} \rightarrow E$. If $v$ is sufficiently close to $h_{n-1} \mid C_{n-1}^{1} \cup A_{n}^{1}$, then $v\left(C_{n-1}^{1} \cup A_{n}^{1}\right) \cap h_{0}\left(F_{1}\right)=\varnothing$. Using the estimated homeomorphism extension property for compacta of $\widetilde{E}$, we extend $v$ to a homeomorphism $h$ of $\widetilde{E}$ with $d^{*}\left(h, h_{n-1}\right)<2^{-n-2}$. We repeat the same argument to $h^{-1} \mid h\left(C_{n}^{1} \cup A_{n}^{1}\right) \cup A_{n}^{2}$ (see the proof of 4.2) to obtain $g$ with $g\left|h\left(C_{n}^{1} \cup A_{n}^{1}\right) \cup A_{n}^{2}=h^{-1}\right| h\left(C_{n}^{1} \cup A_{n}^{1}\right) \cup A_{n}^{2}$ and $d^{*}\left(g, h^{-1}\right)<2^{-n-2}$. Finally, we let $h_{n}=g^{-1}$.

\section{Relative topological EQUiVAlENCE OF SKELETOIDS IN NONCOMPLETE SPACES}

The importance of skeleton sets follows from their relative topological equivalence in complete metric spaces $X$, i.e., if $X_{1}$ and $X_{2}$ are skeletoids then there exists $h \in H(X)$ with $h\left(X_{1}\right)=X_{2}$. This is no longer true if one drops the completeness assumption of $X$. To show this, let us consider the simplest example of a skeletoid, a countable dense subset of a separable m.l.s. $X$ which is a skeleton set for the class of all finite sets of $X$.

5.1. Example. For each $n$, let $E_{n}=\left\{\left(x_{i}\right) \in l_{2}^{f}: x_{i}=0\right.$ for $\left.i \geq n+1\right\}$. Pick a minimal $1 / n$-net $A_{n}$ in $E_{n} \backslash E_{n-1}(n \geq 1)$. Then the set $A=\bigcup_{1}^{\infty} A_{n}$ is a countable dense subset of $l_{2}^{f}$. Let $B$ be a countable dense subset of $l_{2}^{f}$ such that $B \cap E_{1}$ is dense in $E_{1}$. There exists a homeomorphism $h$ of $l_{2}$ carrying $A$ onto $B$. However, there is no homeomorphism $h$ of $l_{2}^{f}$ with $h(A)=B$. This is a consequence of the fact that $l_{2}^{f} \backslash A$ being the union of $\left(E_{n} \backslash E_{n-1}\right) \backslash A_{n}$ is $\sigma$-compact but $l_{2}^{f} \backslash B$ is not. If $l_{2}^{f} \backslash B$ were $\sigma$-compact, then $E_{1} \backslash B$ would be also $\sigma$-compact, contradicting the Baire category theorem.

Let us recall that every m.l.s. spanned by countable many linearly independent vectors is a skeletoid (for the class of finite-dimensional compacta) in its completion [3]. Below we show that two such skeletoids are no longer relatively topological equivalent if they are contained in $\Sigma$, the linear span of the Hilbert space $l_{2}$.

5.2. Example. Consider a linearly independent subset $\left\{x_{i}\right\}_{i=1}^{\infty}$ of $l_{2}$ such that $E_{1}=\operatorname{span}\left\{x_{i}\right\}_{i=1}^{\infty}$ is dense in $l_{2}$ and $E_{1} \cap \Sigma=\{0\}$. Let $\Sigma^{\prime}=\Sigma+E$. Both 
$\Sigma$ and $\Sigma^{\prime}$ are skeletoids for the class of compacta in $l_{2}$; consequently there eixsts $h \in H\left(l_{2}\right)$ with $h(\Sigma)=\Sigma^{\prime}$; see [17]. However, no homeomorphism of $\Sigma$ onto $\Sigma^{\prime}$ sends $E_{0}=l_{2}^{f} \subset \Sigma$ onto $E_{1} \subset \Sigma^{\prime}$. The reason is that every $\Sigma_{n}=\left(\Sigma+\operatorname{span}\left\{x_{1}, x_{2}, \ldots, x_{n}\right\}\right) \backslash \operatorname{span}\left\{x_{1}, x_{2}, \ldots, x_{n}\right\}$ is $\sigma$-compact and so is their union $\Sigma^{\prime} \backslash E_{1}=\bigcup_{1}^{n} \Sigma_{n}$, but $\Sigma \backslash E_{0}$ is not $\sigma$-compact. If $\Sigma \backslash E_{0}$ were $\sigma$ compact, $\left\{\left(x_{i}\right) \in l_{2}:\left|x_{i}\right| \leq 1 / i\right\} \backslash E_{1}$ would also be $\sigma$-compact, contradicting the Baire category theorem.

The following result shows that the $\sigma$-compactness of complements of skeletoids considered in 5.1 suffices to obtain their relative topological equivalence in $l_{2}^{f}$.

5.3. Theorem. Let $A$ and $B$ be countable dense subsets of $l_{2}^{f}$. If $l_{2}^{f} \backslash A$ and $l_{2}^{f} \backslash B$ are $\sigma$-compact, then there exists $h \in H\left(l_{2}\right)$ with $h\left(l_{2}^{f}\right)=l_{2}^{f}$ and $h(A)=$ $B$.

We shall need the following.

5.4. Lemma. Let $A$ be a countable dense subset of $l_{2}^{f}$. Given a pair of finitedimensional compacta $(K, L)$, every map $F:(K, L) \rightarrow\left(l_{2}, l_{2}^{f}\right)$ such that $f \mid L$ is an embedding into $l_{2}^{f} \backslash A$ can be arbitrarily closely approximated by embeddings $v: K \rightarrow l_{2}^{f} \backslash A$ with $v|L=f| L$.

Proof. First, since $l_{2} \backslash l_{2}^{f}$ is locally homotopy negligible in $l^{2}$ [19], we can assume that $f(A) \subset l_{2}^{f}$. Consider any embedding $i: K \rightarrow[0,1]^{q} \times\{0\} \subset$ $[0,1]^{q} \times[0,1]$ for a suitable integer $q$. Pick a map $\alpha:[0,1]^{q} \rightarrow[0,1]$ with $\alpha^{-1}(\{0\})=i(L)$. Write $M=\left\{(p, t) \in[0,1]^{q} \times[0,1]: t \leq \alpha(p)\right\}$ and let $\bar{f}: M \rightarrow l_{2}^{f}$ be given by $\bar{f}(p, t)=f \circ i^{-1}(p)$. By a property of $l_{2}^{f}$ there exists an embedding $\bar{v}: M \rightarrow l_{2}^{f}$ with $\bar{v}|i(L)=\bar{f}| i(L)$ and as close to $\bar{f}$ as we wish [6]. Consider a parametric family of embeddings $v_{t}(k)=\bar{v}(i(k), t \alpha(i(k)))$, $0 \leq t \leq 1, k \in K$. Since for $t \neq s, v_{t}(K \backslash L) \cap v_{s}(K \backslash L)=\varnothing$, for some $t$ we have $v_{t}(K) \cap A=\varnothing$. We set $v=v_{t}$.

In the proof below we slightly modify the technique of skeleton sets. Namely, we deal with two pairs of skeletoids $\left(A, l_{2}^{f} \backslash A\right)$ and $\left(B, l_{2}^{f} \backslash A\right)$, and while $A$ and $B$ are skeletoids for the class of finite sets, $l_{2}^{f} \backslash A$ and $l_{2}^{f} \backslash B$ are skeletoids for the class of finite-dimensional compacta. Our goal is to construct $h \in H\left(l_{2}\right)$ sending $\left(A, l_{2}^{f} \backslash A\right)$ onto $\left(B, l_{2}^{f} \backslash B\right)$.

Proof of 5.3. Let $l_{2}^{f} \backslash A=\bigcup_{1}^{\infty} A_{n}^{1}$ and $l_{2}^{f} \backslash B=\bigcup_{1}^{\infty} A_{n}^{2}$, where $A_{n}^{i}$ are compacta. Let, moreover, $A=\left\{a_{n}^{1}\right\}_{n=2}^{\infty}$ and $B=\left\{a_{n}^{2}\right\}_{n=1}^{\infty}$. Inductively, we shall construct sequences of finite-dimensional compacta $\left\{C_{n}^{1}\right\}$ of $l_{2}^{f} \backslash A$ and $\left\{C_{n}^{2}\right\}$ of $l_{2}^{f} \backslash B$ finite sets $F_{n}^{1} \subset A$ and $F_{n}^{2} \subset B$, and a sequence $\left\{h_{n}\right\}_{1}^{\infty}$ of homeomorphisms of $l_{2}$ such that for $n \geq 2$

(1) $A_{n}^{1} \cup\left\{a_{i}^{1}\right\}_{i=2}^{n} \subset C_{n}^{1} \cup F_{n}^{1}$ and $A_{2}^{n} \cup\left\{a_{i}^{2}\right\}_{i=2}^{n} \subset h_{n}\left(C_{n}^{1} \cup F_{n}^{1}\right) \subset C_{n}^{2} \cup F_{n}^{2}$; 
(2) $h_{n}\left|C_{n-1}^{1} \cup F_{n-1}^{1}=h_{n-1}\right| C_{n-1}^{1} \cup F_{n-1}^{1}$;

(3) $d^{*}\left(h_{n}, h_{n-1}\right)=d\left(h_{n}, h_{n-1}\right)+d\left(h_{n}^{-1}, h_{n-1}^{-1}\right) \leq 2^{-n}$.

Then, clearly $h=\lim h_{n}$ will be a required homeomorphism.

Inductive construction. With $A_{1}^{i}=C_{1}^{i}=F_{1}^{i}=\varnothing(i=1,2)$ we may set $h_{1}=$ id. Assume that $h_{1}, h_{2}, \ldots, h_{n-1}(n \geq 2)$ exist. By 5.4, $h_{n-1} \mid C_{n-1}^{1} \cup A_{n}^{1}$ can be approximated by an embedding $v: C_{n-1}^{1} \cup A_{n}^{1} \rightarrow l_{2}^{f} \backslash B$ with $v \mid C_{n-1}^{1}=$ $h_{n-1} \mid C_{n-1}^{1}$. We pick a point $v\left(a_{n}^{1}\right) \in B$ as close to $h_{n-1}\left(a_{n}^{1}\right)$ as we wish and such that $\left(h_{n-1} \mid F_{n}^{1}\right) \cup v\left(a_{n}^{1}\right)$ is one-to-one. By the estimated homeomorphism extension property applied to $l_{2}$, there is $h \in H\left(l_{2}\right)$ which extends $v$, i.e., $h\left(C_{n-1}^{1} \cup A_{n}^{1}\right) \subset l_{2}^{f} \backslash B, h\left(F_{n-1}^{1} \cup\left\{a_{n}^{1}\right\}\right) \subset B, h\left|C_{n-1}^{1} \cup F_{n-1}^{1}=h_{n-1}\right| C_{n-1}^{1} \cup$ $F_{n-1}^{1}$ and $d^{*}\left(h, h_{n-1}\right)<2^{-n-2}$. Next, applying 5.4, $h^{-1} \mid h\left(C_{n-1}^{1} \cup A_{n}^{1}\right) \cup A_{n}^{2}$ can be approximated by an embedding $v$ into $l_{2}^{f} \backslash A$ with $v \mid h\left(C_{n-1}^{1} \cup A_{n}^{1}\right)=$ $h^{-1} \mid h\left(C_{n-1}^{1} \cup A_{n}^{1}\right)$. We can find a point $v\left(a_{n}^{2}\right) \in A$ as close to $h^{-1}\left(a_{n}^{2}\right)$ as we wish and such that $h^{-1} \mid h\left(F_{n}^{1} \cup\left\{a_{n}^{1}\right\}\right) \cup v\left(a_{n}^{2}\right)$ is one-to-one. We extend $v$ to a homeomorphism $g$ of $l_{2}$ with $d^{*}\left(g, h^{-1}\right)<2^{-n-2}$. Finally, we set $C_{n}^{1}=C_{n-1}^{1} \cup A_{n}^{1} \cup g^{-1}\left(A_{n}^{2}\right), C_{n}^{2}=h\left(C_{n-1}^{1} \cup A_{n}^{1}\right) \cup A_{n}^{2}, F_{n}^{1}=F_{n}^{1} \cup\left\{a_{n}^{1}\right\} \cup\left\{g^{-1}\left(a_{n}^{2}\right)\right\}$, $F_{n}^{2}=h\left(F_{n}^{1} \cup\left\{a_{n}^{1}\right\}\right) \cup\left\{a_{n}^{2}\right\}$ and $h_{n}=g^{-1}$.

5.5. Remark. The assertion of 5.3 holds true if one replaces $l_{2}^{f}$ by $\Sigma$. An argument of 5.3 can be adopted to prove the following fact.

5.6. Proposition. Let $E$ be an infinite-dimensional $\sigma$-compact l.c.m.l.s. and let $E_{1}$ and $E_{2}$ be two homeomorphic dense linear subspaces of $E$ with $\sigma$-compact complements. Suppose that $E \backslash E_{i}$ can be represented as a union of compacta, $E \backslash E_{i}=A_{n}^{i}$, in such a way that each $A_{n}^{1}$ embeds in $E \backslash E_{2}$ and each $A_{n}^{2}$ embeds in $E \backslash E_{1}$. Then, there exists a homeomorphism $h$ of the completion $\check{E}$ of $E$ with $h(E)=E$ and $h\left(E_{1}\right)=E_{2}$.

We need the following modification of 4.3.

5.7. Lemma. Let $E$ be an infinite-dimensional l.c.m.l.s. and $E_{0}$ its dense linear subspace. Let $(A, B)$ be a pair of compacta such that $A$ embeds in $E \backslash E_{0}$. Given a compact set $K, \varepsilon>0$ and a map $f:(A, B) \rightarrow\left(\check{E}, E \backslash E_{0}\right)$ such that $f \mid B$ is an embedding, there exists an embedding $v: A \rightarrow E \backslash E_{0}$ with $v|B=f| B$, $d(v, f)<\varepsilon$ and $v(A \backslash B) \cap K=\varnothing$.

Proof. We may assume that $f(A) \subset E$ (see the proof of 4.3) and $A \subset E \backslash E_{0}$ with $A \cap(f(A) \cup K)=\varnothing$. Let $A \backslash B=\bigcup_{1}^{\infty} A_{i}$, where $\left\{A_{i}\right\}$ is a tower of compacta. Applying 2.6, we construct a sequence $\left\{h_{n}\right\}_{0}^{\infty} \subset H\left(E \mid E_{0}\right)$ such that (1)-(3) of 4.3 are satisfied. As in the proof of $4.3 v=\lim h_{n} \mid A$ is an embedding with $v|B=f| B$ and $v(A \backslash B) \cap K=\varnothing$. Since $A_{n} \subset E \backslash E_{0}$ and $h_{n}$ preserves $E_{0}, v\left(A_{n}\right)=h_{n}\left(A_{n}\right) \subset E \backslash E_{0}(n=1,2, \ldots)$. 
Proof of 5.3. Let $E_{1}=\bigcup_{1}^{\infty} B_{n}^{1}, E_{2}=\bigcup_{1}^{\infty} B_{n}^{2}$, where $B_{n}^{i}$ are compacta. We construct sequences of compacta $\left\{C_{n}^{1}\right\}$ of $E \backslash E_{1},\left\{C_{n}^{2}\right\}$ of $E \backslash E_{2},\left\{D_{n}^{1}\right\}$ of $E_{1}$ and $\left\{D_{n}^{2}\right\}$ of $E_{2}$ and a sequence of homeomorphisms $\left\{h_{n}\right\}_{n=1}^{\infty}$ of $\check{E}$ such that for $n \geq 2$

(1) $A_{n}^{1} \cup B_{n}^{1} \subset C_{n}^{1} \cup D_{n}^{1}$ and $A_{n}^{2} \cup B_{n}^{2} \subset h_{n}\left(C_{n}^{1} \cup D_{n}^{1}\right) \subset C_{n}^{2} \cup D_{n}^{2}$;

(2) $h_{n}\left|C_{n-1}^{1} \cup D_{n-1}^{1}=h_{n-1}\right| C_{n-1}^{1} \cup D_{n-1}^{1}$;

(3) $d^{*}\left(h_{n}, h_{n-1}\right)<2^{-n}$.

We omit the inductive construction. To perform it, follow the proof of 4.2 and make use of the result of 5.5. We complete the proof by letting $h=\lim h_{n}$.

5.8. Theorem. Let $E$ be a $\sigma$-compact l.c.m.l.s. containing a Hilbert cube (by a result of [10] $E$ is homeomorphic to $\Sigma$ ). Let $E_{1}$ and $E_{2}$ be homeomorphic $\sigma$-compact dense linear subspaces of $E$. If $E_{i}$ contains no Hilbert cube and $E \backslash E_{i}(i=1,2)$ are $\sigma$-compact, then there exists a homeomorphism $h$ of the completion $\check{E}$ of $E$ such that $h(E)=E$ and $h\left(E_{1}\right)=E_{2}$.

Proof. Let $Q$ denote a Hilbert cube contained in $E$. Since each $Q \backslash E_{i}$ is nonempty and $\sigma$-compact, by the Baire category theorem, it contains an open subset of $Q$. Consequently, each $E \backslash E_{i}$ is universal for compacta. Therefore, the assertion follows from 5.6.

A direct conclusion of 5.8 is the following.

5.9. Corollary. Let $E_{1}$ and $E_{2}$ be dense linear subspaces which are countable unions of finite-dimensional compacta of $\Sigma$. If $E \backslash E_{i}$ are $\sigma$-compact $(i=1,2)$, then there exists a homeomorphism of $l_{2}$ with $h(\Sigma)=\Sigma$ and $h\left(E_{1}\right)=E_{2}$.

5.10. Note. If, additionally in 5.9, $A \subset E_{1}$ and $B \subset E_{2}$ are countable and dense sets such that both $E_{1} \backslash A$ and $E_{2} \backslash B$ are $\sigma$-compact, then there exists a homeomorphism $h$ of $l_{2}$ with $h(\Sigma)=\Sigma, h\left(E_{1}\right)=E_{2}$ and $h(A)=B$.

In the case where the complements $E \backslash E_{i}$ are not $\sigma$-compact, we have the following partial result only.

5.11. Theorem. Let $E$ be a l.c.m.l.s. such that $E=\bigcup_{1}^{\infty} C_{n}$, where each $C_{n}$ is a compact convex set and $C_{n}$ is a $Z$-set in $C_{n+1}$. Let $E_{1}$ and $E_{2}$ be dense linear subspaces which are countable unions of finite-dimensional compacta of $E$. If each $C_{n} \cap E_{i}(i=1,2)$ is dense in $C_{n}$, then there exists a homeomorphism $h$ of the completion $\check{E}$ of $E$ such that $h(E)=E$ and $h\left(E_{1}\right)=E_{2}$.

We start with a lemma.

5.12. Lemma. Let $C_{1} \subset C_{2} \subset \cdots \subset C_{k}$ be a tower of compact convex sets in an infinite-dimensional l.c.m.l.s. $E$. Let $E_{0}$ be a linear dense subspace of $E$ with each $C_{i} \cap E_{0}$ dense in $C_{i}$ and assume $C_{i}$ is a $Z$-set in $C_{i+1}, i=1, \ldots, k-1$. Then, every embedding of a pair of finite-dimensional subcompacta $(A, B)$ of $C_{k} u:(A, B) \rightarrow\left(C_{k}, C_{k} \cap E_{0}\right)$ with $u\left(A \cap\left(C_{i} \backslash C_{i-1}\right)\right) \subset C_{i} \backslash C_{i-1}\left(C_{0}=\varnothing\right)$ can be arbitrarily closely approximated by embeddings $v: A \rightarrow C_{k}$ with $v\left(A \cap C_{1}\right) \subset$ $E_{0}, v|B=u| B$ and $v\left(A \cap\left(C_{i} \backslash C_{i-1}\right)\right) \subset C_{i} \backslash C_{i-1}$. 
Proof. By virtue of [9], a convex set $C_{1} \cap E_{0}$ is homeomorphic to $l_{2}^{f}$. Hence, by a property of $l_{2}^{f}, u \mid A \cap C_{1}$ can be approximated by embeddings $v_{1}: A \cap C_{1} \rightarrow$ $C_{1} \cap E_{0}$ such that $v_{1}\left|B \cap C_{1}=u\right| B \cap C_{1}$. There is a map $f_{1}: A \cap C_{2} \rightarrow C_{2}$ with $f_{1}\left|A \cap C_{1}=v_{1}\right| A \cap C_{1}$ and $f_{1}\left|B \cap C_{2}=u\right| B \cap C_{2}$. We can find $f_{1}$ as close to $u \mid A \cap C_{2}$ as we wish, provided $v_{1}$ is close enough to $u \mid A \cap C_{1}$. Consider $C_{2} \cap E_{0}$, another copy of $l_{2}^{f}$. Since $C_{1} \cap E_{0}$ is a $Z$-set in $C_{2} \cap E_{0}$, the map $f_{1}$ can be approximated by embeddings $v_{2}: A \cap C_{2} \rightarrow C_{2} \cap E_{0}$ satisfying $v_{2}\left(A \cap\left(C_{2} \backslash C_{1}\right)\right) \cap C_{2} \backslash C_{1}$ and $v_{2}\left|\left(A \cap C_{1}\right) \cup\left(B \cap C_{2}\right)=f_{1}\right|\left(A \cap C_{1}\right) \cup\left(B \cap C_{2}\right)$. Continuing this process, we find embeddings $v_{i}: A \cap C_{i} \rightarrow C_{i} \cap E_{0}$ such that $v_{i}\left|A \cap C_{i-1}=v_{i-1}\right| A \cap C_{i-1}, v_{i}\left(A \cap\left(C_{i} \backslash C_{i-1}\right)\right) \subset C_{i} \backslash C_{i-1}$ and $v_{i}$ to be as close to $u \mid A \cap C_{i}$ as we wish. Clearly, $v=v_{k}$ fulfills the assertion of 5.12 .

Proof of 5.11. It is easily seen that there are sequences of finite-dimensional compacta $\left\{A_{n}^{1}\right\}_{1}^{\infty}$ and $\left\{A_{n}^{2}\right\}_{1}^{\infty}$ with $A_{n}^{i} \subset C_{n}$ and such that $\bigcup_{1}^{\infty} A_{n}^{i}=E_{1}, i=$ 1,2 . We shall construct inductively sequences of finite-dimensional compacta $\left\{C_{n}^{i}\right\}_{1}^{\infty}$, a sequence of integers $1=k(1)<k(2)<\cdots$, and a sequence of homeomorphisms $\left\{h_{n}\right\}_{1}^{\infty}$ of $E$ such that for $n \geq 2$,

(1) $A_{n}^{1} \subset C_{n}^{1} \subset C_{k(n)}$ and $A_{n}^{2} \subset h_{n}\left(C_{n}^{1}\right) \subset C_{n}^{2} \subset C_{k(n)}$;

(2) $h_{n}\left|C_{n-1}^{1}=h_{n-1}\right| C_{n-1}^{1}$;

(3) $h_{n}\left(D_{k(i)}\right)=D_{k(i)}$ with $D_{j}=C_{j} \backslash C_{j-1}, i=1, \ldots, n$;

(4) $d^{*}\left(h_{n}, h_{n-1}\right)=d\left(h_{n}, h_{n-1}\right)+d\left(h_{n}^{-1}, h_{n-1}^{-1}\right)<2^{-n}$.

Suppose $\left\{h_{n}\right\}_{1}^{\infty}$ has been constructed. Then, by (4), $h=\lim h_{n}$ is a homeomorphism of $\check{E}$ satisfying, by (1) and (2), $h\left(E_{1}\right)=E_{2}$. Condition (3) implies $h\left(C_{k(i)}\right)=C_{k(i)}$ for every $i$. Therefore, we additionally obtain $h(E)=E$.

Inductive construction. To construct $h_{1}$, consider $C_{1} \cap E_{2}$, a convex copy of $l_{2}^{f}$; see [8]. Since $C_{1} \cap E_{2}$ is dense in $C_{1}$, the identity on $A_{1}^{1}$ can be approximated by embeddings $v: A_{1}^{1} \rightarrow C_{1} \cap E_{2}$. The estimated homeomorphism extension property [4, p. 155] of $C_{1}$, homeomorphic to the Hilbert cube [4, p. 100], implies that the identity on $C_{1}$ can be approximated by homeomorphisms $\bar{h}$ of $C_{1}$ with $\bar{h}\left|A_{1}^{1}=v\right| A_{1}^{1}$. Next, we extend $\bar{h}$ to a homeomorphism $h$ of $\check{E}$; moreover, the estimated homeomorphism extension property of $\check{E}$ yields $h$ with $d^{*}(h$, id $)<1 / 4$ if $\bar{h}$ is sufficiently close to $\operatorname{id}_{C_{1}}$. Let $A=h\left(A_{1}^{1}\right) \cup A_{1}^{2}$ and $B=h\left(A_{1}^{1}\right)$, and let $u=h^{-1} \mid A$. Now $C_{1} \cap E_{1}$ is a dense convex copy of $l_{2}^{f}$ in $C_{1}$. Hence, $u$ can be approximated by embeddings $v: A \rightarrow C_{1} \cap E_{1}$ with $v|B=u| B$. As above, we extend $v$ to a homeomorphism $g$ of $\breve{E}$ satisfying $g\left(C_{1}\right)=C_{1}$ and $d^{*}\left(g, h^{-1}\right)<1 / 4$. We set $h_{1}=g^{-1}$ together with $C_{1}^{1}=$ $A_{1}^{1} \cup g\left(A_{1}^{2}\right)$ and $C_{1}^{2}=h\left(A_{1}^{1}\right) \cup A_{1}^{2}$.

Assume that (1)-(4) are valid for $n \geq 2$. Consider $A=C_{n}^{1} \cup A_{n+1}^{1}, B=C_{n}^{1}$, and $u=h_{n} \mid A$. There exists an integer $k(n+1)>k(n)$ and an embedding $v_{0}: A \rightarrow C_{k(n+1)}$ as close to $u$ as we wish with $v_{0}\left|A \cap C_{k(n)}=u\right| A \cap C_{k(n)}$. 
Applying 5.12 to a tower $C_{k(1)} \subset C_{k(2)} \subset \cdots \subset C_{k(n+1)}$, we find an embedding $v: A \rightarrow C_{k(n+1)}$ such that $v(A) \subset E_{2}, v\left|B=v_{0}\right| B=h_{n} \mid B$, and $v\left(A \cap D_{k(i)}\right) \subset$ $D_{k(i)}, i=1,2, \ldots, n+1$. Since each $C_{k(i)}$ is a $Z$-set in $C_{k(i+1)}$ and $C_{k(i+1)}$ is a $Z$-set in $\check{E}$, the estimated homeomorphism extension properties of each $C_{k(i+1)}$ and $\check{E}$ yield that there exists a homeomorphism $h$ of $\check{E}$ preserving each $C_{k(i)}, i=1,2, \ldots, n+1$, and satisfying $h|A=v| A$ and $d^{*}\left(h, h_{n}\right)<2^{-n-2}$. Next, let $A=h\left(C_{n}^{1} \cup A_{n+1}^{1}\right) \cup A_{n+1}^{2}, B=h\left(C_{n}^{1} \cup A_{n+1}^{1}\right)$ and $u=h^{-1} \mid A$. We have $A \subset C_{k(n+1)}$ and $u\left(A \cap D_{k(i)}\right) \subset D_{k(i)}, i=1,2, \ldots, n+1$. Applying 5.12, we approximate $u$ by an embedding $v: A \rightarrow C_{k(n+1)}$ with $v(A) \subset E_{1}$ and $v\left(A \cap D_{k(i)}\right) \subset D_{k(i)}$ for $i=1,2, \ldots, n+1$. Finally, we extend $v$ in the above fashion to a homeomorphism $g$ of $\check{E}$ which preserves each $C_{k(i)}, i=$ $1,2, \ldots, n+1$, and $d^{*}\left(g, h^{-1}\right)<2^{-n-2}$. To finish the inductive construction we set $h_{n+1}=g^{-1}, C_{n+1}^{1}=C_{n}^{1} \cup A_{n+1}^{1} \cup g^{-1}\left(A_{n+1}^{2}\right), C_{n+1}^{2}=h\left(C_{n}^{1} \cup A_{n+1}^{1}\right) \cup A_{n+1}^{2}$.

5.13. Note. The proof of 5.11 works in the case where $\bigcup_{1}^{\infty} C_{i}=C$ is a nontrivial convex subset of $E$. If $C^{1}\left(=E_{1}\right)$ and $C^{2}\left(=E_{2}\right)$ are convex subsets of $C$ and each $C_{k} \cap C^{i}$ is $\sigma$-finite-dimensional dense in $C$, then there exists a homeomorphism $h$ of the closure $\bar{C}$ of $C$ with $h(C)=C$ and $h\left(C^{1}\right)=C^{2}$ (see [6]).

5.14. Example. Let $Q=[-1,1]^{N}$ be the Hilbert cube. Consider $C=$ $\bigcup_{1}^{\infty}\left\{\left(x_{i}\right) \in Q:\left|x_{i}\right|<1-\frac{1}{n}, i \geq n+1\right\}$, a convex dense subset of $Q$, and let $I_{n}=\left\{\left(x_{i}\right) \in Q: x_{i}=0, i \geq n+1\right\}$ and $\stackrel{\circ}{I}_{n}=\left\{\left(x_{i}\right) \in I_{n}:\left|x_{i}\right|<1, i=\right.$ $1,2, \ldots, n\}$. We infer from 5.13 that there exists $h \in H(Q)$ with $h(C)=C$ and $h\left(\bigcup_{n} I_{n}\right)=\bigcup_{n} \stackrel{\circ}{I}_{n}$.

\section{EXTENDING HOMEOMORPHISMS IN AN ISOTOPY SETTING}

It is possible to give an isotopy version of our abstract theorem concerning the homeomorphism extension property. To obtain that both notions, the almost extension and the limit flattening properties, have to be changed. We are not going to present this in full generality. We shall only discuss the case of extending homeomorphisms in locally convex metric linear spaces. Our controlled isotopy extension theorems are formulated in a similar way to that of AndersonMcCharen [2]. The results, as the referee suggested, may have applications in investigating manifolds modelled on metric linear spaces without completeness. We include here, as a direct application of that, the homeomorphism extension theorem for local compacta in such spaces.

Before we formulate a controlled isotopy extension theorem we explain the following notation concerning homotopies and isotopies. We say that a homotopy $h=\left(h_{t}\right): Z \rightarrow E$ is limited by a collection $\mathscr{U}$ of subsets of $E$ if each set $h(z \times I), z \in Z$, is either contained in some member of $\mathscr{U}$ or it consists of one point. We write $I$ for the interval $[0,1]$ and $\mathscr{U}^{+}=\bigcup \mathscr{U}$. Together with a 
homotopy $\left(h_{t}\right), h_{0}=$ id, we shall consider the set Fix $(h)=\{z \in Z: h(z \times I)=$ $\{z\}\}$. Clearly we have $\mathscr{U}^{+} \supset h((Z \backslash \operatorname{Fix}(h)) \times I)$. By an isotopy $\bar{h}=\left(\bar{h}_{t}\right)$ of $E$ we mean a homotopy $\bar{h}: E \times I \rightarrow E$ with the property that $(x, t) \rightarrow(\bar{h}(x, t), t)$ is a homeomorphism of $E \times I$.

6.1. Theorem. Let $E$ be an infinite-dimensional l.c.m.l.s. and let $K$ be its compact subset. Let $h=\left(h_{t}\right)$ be a homotopy of $K$ into $E$ so that $h_{0}=\mathrm{id}_{K}$ and $h_{1}: K \rightarrow E$ is an embedding. Assume that $h$ is limited by a collection $\mathscr{U}$ of open subsets of $E$. If $\mathscr{U}^{+}$contains the closure of $K \backslash \operatorname{Fix}(h)$ then there exists an isotopy $\bar{h}=\left(\bar{h}_{t}\right)$ of $E$ such that $\bar{h}_{0}=\mathrm{id}, \bar{h}_{1} \mid K=h_{1}, \bar{h}$ is limited by $\mathscr{U}$ and $\bar{h}(k, t)=k$ for all $k \in \operatorname{Fix}(h)$ and $t \in I$.

First we modify the homotopy $h$ in order to have $h((K \backslash \operatorname{Fix}(h)) \times I) \cap$ $\operatorname{Fix}(h)=\varnothing$.

6.2. Lemma. Write $K_{0}=\operatorname{Fix}(h)$. There exists a homotopy $g=\left(g_{1}\right)$ of $K$ which is limited by $\mathscr{U}$ and such that $g=h$ on $K_{0} \times I \cup\left(K \backslash K_{0}\right) \times\{0,1\}$ and $g\left(\left(K \backslash K_{0}\right) \times I\right) \cap K_{0}=\varnothing$.

Proof. Write $\bar{\varepsilon}(k)=\frac{1}{2} \sup \{\operatorname{dist}(h(k \times I), E \backslash U): U \in \mathscr{U}\}$ and notice that $\bar{\varepsilon}$ is lower-semicontinuous on $K \cap \mathscr{U}^{+}$. There exists a continuous mapping $\varepsilon$ with $0<\varepsilon(k) \leq \bar{\varepsilon}(k), k \in K \cap \mathscr{U}^{+}$. Correct $\varepsilon$ to have $\varepsilon\left(k_{n}\right) \rightarrow 0$ whenever $d\left(k_{n}, K_{0}\right) \rightarrow 0$. Since $K_{0}$ is a $Z$-set in $E$, one can find a homotopy $\phi=\left(\phi_{s}\right)$ on $\left(K \backslash K_{0}\right) \times I$ into $I$ so that $\phi_{s}\left(\left(K \backslash K_{0}\right) \times I\right) \cap K_{0}=\varnothing$ for all $0<s \leq 1, \phi_{0}=h$ on $\left(K \backslash K_{0}\right) \times I$ and $d\left(\phi_{s}(k, t), h(k, t)\right)<\varepsilon(k)$ for all $k \in K \backslash K_{0}$ and $(t, s) \in I \times I$, see [19]. The latter inequality yields: $d\left(\phi_{s}(k, t), h(k, t)\right)<\operatorname{dist}(h(k \times I), E \backslash U)$ for some $U \in \mathscr{U}$ containing $h(k \times I)$; i.e., $\phi_{s}(k, t) \in U$ for $(s, t) \in I \times I$. Pick a steering map $\lambda:\left(K \backslash K_{0}\right) \times I \rightarrow I$ such that $\lambda^{-1}(0) \in\left(K \backslash K_{0}\right) \times\{0,1\}$ and put $g(k, t)=\phi((k, t), \lambda(k, t))$ for $(k, t) \in\left(K \backslash K_{0}\right) \times I$. It is clear that this map can be continuously extended to the whole $K \times I$ by putting $g=h$ on $K_{0} \times I$. Now we show that $\mathscr{U}$ can be replaced by a collection $\left\{\operatorname{st}(h(k \times I), \mathscr{V}): k \in K \cap \mathscr{U}^{+}\right\}$ for some collection $\mathscr{V}$; here $\operatorname{st}(A, \mathscr{V})$ stands for $\bigcup\{V \in \mathscr{V}: V \cap A=\varnothing\}$.

6.3. Lemma. There exists a collection $\mathscr{V}$ consisting of open sets of $E$ such that $\mathscr{V}^{+}$contains $h\left(\left(K \cap \mathscr{U}^{+}\right) \times I\right)$ and $\mathrm{st}((k \times I), \mathscr{V})$ is contained in some element of $\mathscr{U}$ for each $k \in K \cap \mathscr{U}^{+}$.

Proof. Let $\varepsilon$ be a function of the proof of 6.2. Write $B(x, r)=\{y: d(x, y)<$ $r\}$. We shall pick for each $x \in h\left(\left(K \cap \mathscr{U}^{+}\right) \times I\right)$ a number $\delta(x)>0$ such that whenever $y \in \bigcup\{B(x, \delta(x)): B(x, \delta(x)) \cap h(k \times I)=0\}$ then

$$
d(y, h(k \times I))<\varepsilon(k) .
$$

From this it easily follows that $\mathscr{V}=\left\{B(x, \delta(x)): x \in h\left(\left(K \cap \mathscr{U}^{+}\right) \times I\right)\right\}$ has the required property. Write $\bar{\delta}(x)=\inf \left\{\frac{1}{4} \varepsilon(k): x \in h(k \times I)\right\}$. By the compactness of $K$ we infer that $\bar{\delta}$ is lower-semicontinuous and $\bar{\delta}(x)>0$ for $x \in$ $h\left(\left(K \cap \mathscr{U}^{+}\right) \times I\right)$. Thus there exists a continuous map $\bar{\delta}$ such that $0<\bar{\delta}(x) \leq$ $\bar{\delta}(x)$. Now, by the continuity of $\bar{\delta}$ there is a continuous map $\delta$ such that 
$0<\delta(x) \leq \overline{\bar{\delta}}(x)$ and that $d(y, x)<2 \delta(x)$ implies $|\bar{\delta}(y)-\overline{\bar{\delta}}(x)|<\overline{\bar{\delta}}(x)$. Assume now that $y \in B(x, \delta(x)), x \in h\left(\left(K \cap \mathscr{U}^{+}\right) \times I\right)$ with $B(x, \delta(x)) \cap$ $h(k \times I)=0$. Then for some $t \in I$ we have $d\left(x, h_{t}(k)\right)<\delta(x)$, and consequently $\left|\bar{\delta}(x)-\bar{\delta}\left(h_{t}(k)\right)\right|<\overline{\bar{\delta}}\left(h_{t}(k)\right)$. This yields $\bar{\delta}(x) \leq 2 \overline{\bar{\delta}}\left(h_{t}(k)\right)$. We can estimate $d\left(y, h_{t}(k)\right) \leq d(y, x)+d\left(x, h_{t}(k)\right) \leq 2 \delta(x) \leq 4 \bar{\delta}\left(h_{t}(k)\right) \leq \varepsilon(k)$. A proof of 6.3 is completed.

The following fact will be our main step in proving 6.1. It replaced the condition of the almost extension property.

6.4. Proposition. Let $\left(K, K_{0}\right)$ be a pair of compacta of $E, U$ an open neighborhood of $K \backslash K_{0}$ in $E$ and $\varepsilon>0$. Suppose $h=\left(h_{t}\right)$ is a homotopy of $K$ in $K_{0} \cup U$ such that $h_{0}=\mathrm{id}, h_{1}: K \rightarrow E$ is an embedding, $h\left(\left(K \backslash K_{0}\right) \times I\right) \cap K_{0}=\varnothing$ and $h_{t} \mid K_{0}=\mathrm{id}$ for all $t \in I$. Then for every collection $\mathscr{V}$ of open subsets of $E$ with $\mathscr{V}^{+} \supset h\left(\left(K \backslash K_{0}\right) \times I\right)$ there exists an isotopy $\left(\phi_{t}\right)$ of $E$ satisfying

(1) $\phi_{0}=$ id,$d\left(\phi_{1} \mid K, h_{1}\right)<\varepsilon$,

(2) $\phi_{t} \mid K_{0} \cup(E \backslash U)=$ id for all $t \in I$,

(3) $\left(\phi_{t}\right)$ is limited by the collection $\left\{\mathrm{st}(h(k \times I), \mathscr{V}): k \in K \backslash K_{0}\right\}$.

6.5. Lemma. The assertion of 6.4 holds true if we additionally assume that $K_{0}=\varnothing$.

Proof. Let $\delta(x)$ be the map of 6.3 chosen for the collection $\mathscr{U}=$ $\{\operatorname{st}(h(k \times I), \mathscr{V}): k \in K\}$. There is a $\delta>0$ so that $\delta \leq \delta(x)$ for all $x \in$ $h(K \times I)$. Thus $\mathscr{U}$ may be replaced by $\{B(h(k \times I), \delta): k \in K\}$, where $B(A, \delta)=\{x \in E: d(x, A)<\delta\}$. A required isotopy $\left(\phi_{t}\right)$ will be given as the composition of an isotopy $\left(\Lambda_{t}\right), 0 \leq t<t_{0}$, such that $\Lambda_{0}=$ id with another isotopy $\left(h_{t}^{\prime}\right), t_{0} \leq t \leq 1$ with $h_{t_{0}}^{\prime}=\mathrm{id}$. Observe that whenever $\left(\Lambda_{t}\right)$ is a $\delta / 2$-isotopy and $\left(h_{t}^{\prime}\right)$ is an isotopy limited by $\{B(h(k \times I), \delta / 2): k \in K\}$ then the isotopy $\left(\phi_{t}\right)$ equal to $\left(\Lambda_{t}\right)$ for $0 \leq t \leq t_{0}$ and $\phi_{t}=h_{t}^{\prime} \circ \Lambda_{t_{0}}$ for $t_{0} \leq t \leq 1$ will be limited by $\{B(h(k \times I), \delta): k \in K\}$. The isotopy $\left(\Lambda_{t}\right)$ that we choose is that of the proof of 2.3. We recall that $\Lambda_{0}=$ id and $\Lambda_{t} \mid E \backslash U=\mathrm{id}, t \in I$. The isotopy $\left(h_{t}^{\prime}\right)$ is given by $h_{t}^{\prime}=h_{0}^{-1} \circ \Phi_{t} \circ h_{0}$, where $h_{0}$ and $\Phi=\left(\Phi_{t}\right)$ are those from the proof of 2.1. We recall that $h_{t}^{\prime}(x)=\alpha \kappa(x)+\Phi(x-\alpha \kappa(x), t \lambda \kappa(x))$, where $\alpha, \kappa$ and $\lambda$ are those of 2.1 and $\Phi$ is an isotopy of a finite-dimensional linear subspace $X_{1}$ of $E$. We additionally require that $\left(h_{t}^{\prime}\right)$ be limited by $\{B(h(k \times I), \delta / 4): k \in K\}$ and $\lambda(y)=0$ for $d(\alpha(y), 0) \geq \delta / 4$. This yields that $\left(h_{t}^{\prime}\right)$ is limited by $\{B(h(k \times I), \delta / 2): k \in K\}$. Both isotopies $\left(\Lambda_{t}\right)$ and $\left(h_{t}^{\prime}\right)$ are taken in such a way that suitable estimates of the proof of 1.5 can be carried over in order to get $d\left(\phi_{1} \mid K, h_{1}\right)<\varepsilon$.

Proof of 6.4. Using the condition that $h\left(\left(K \backslash K_{0}\right) \times I\right) \cap K_{0}=\varnothing$ we infer that for every neighborhood $U_{0}$ of $K_{0}$ in $K$ there exists an open neighborhood $V_{0}$ of $K_{0}$ such that $k^{\prime} \in U_{0}$ provided $k \in V_{0}$ and $d\left(k, h_{t}\left(k^{\prime}\right)\right)<\delta$. Consider $U_{0}=\left\{k \in K: d\left(h_{t}(k), k\right)<\varepsilon / 4, t \in I\right\}$ and pick $V_{0} \subseteq U_{0}$ and $\delta>0, \delta<$ $\varepsilon / 4$, to fulfill the above condition. With $L=K \backslash V_{0},\left(h_{t}\right) \mid L$ and $\{B(x, \delta(x))$ : 
$x \in h(L \times I)\}$ apply 6.5 to get an isotopy $\left(\phi_{t}\right)$ of $E$ such that $\phi_{0}=$ id, $d\left(\phi_{1}\left|L, h_{1}\right| L\right)<\varepsilon / 2, \phi_{t} \mid(E \backslash U) \cup K_{0}=$ id for all $t \in I$ and $\left(\phi_{t}\right)$ is limited by $\{B(h(k \times I), \delta / 2): k \in L\}$. We may assume that $\delta$ is so small that $\{B(h(k \times I), \delta): k \in L\}$ refines the collection $\left\{\operatorname{st}(h(k \times I), \mathscr{V}): k \in K \backslash K_{0}\right\}$. Therefore it is enough to check that $d\left(\phi_{1} \mid K, h_{1}\right)<\varepsilon$. Pick $k \in V_{0} \backslash K_{0}$. Then $\phi(K \times I)$ is contained in $B\left(h\left(k^{\prime} \times I\right), \delta / 2\right)$ for some $k^{\prime} \in L$ and thus $d\left(k, h_{t}\left(k^{\prime}\right)\right)<\delta / 2$. By the choice of $\delta$ we infer that $k^{\prime} \in U_{0}$ which implies that $\operatorname{diam}\left(h\left(k^{\prime} \times I\right)\right)<\varepsilon / 2$. Finally we have

$$
\begin{aligned}
d\left(\phi_{1}(k), h_{1}(k)\right) & \leq d\left(\phi_{1}(k), k\right)+d\left(k, h_{1}(k)\right) \\
& \leq \operatorname{diam}\left(h\left(k^{\prime} \times I\right)\right)+\delta+\varepsilon / 4<\varepsilon .
\end{aligned}
$$

The following consequence of Proposition 6.4 corresponds to the condition $(*)$ of 1.2 .

6.6. Corollary. For every pair of compacta $\left(K, K_{0}\right)$ of $E$, a neighborhood $U$ of the closure of $K \backslash K_{0}$ and a map $\varepsilon: U \rightarrow(0, \infty)$ there exists $\alpha>0$ such that every embedding $h: K \rightarrow E$ with $h \mid K_{0}=\mathrm{id}$ and $d(h$, id $)<\alpha$ can be arbitrary closely approximated by restrictions $\bar{h}_{1} \mid K$, where $\left(\bar{h}_{t}\right)$ is an isotopy of $E$ such that $\bar{h}_{0}=\mathrm{id}, \bar{h}_{t} \mid K_{0} \cup(E \backslash U)=\mathrm{id}$ and $d\left(\bar{h}_{t}(x), x\right)<\varepsilon(x)$ for all $x \in U$ and $t \in I$.

We shall employ the following simple fact.

6.7. Lemma. Suppose that an isotopy $\left(\phi_{t}\right)$ of $E, \phi_{0}=\mathrm{id}$, is limited by $\left\{\mathbf{s t}(h(K \times I), \mathscr{W}): k \in K \backslash K_{0}\right\}$, where $\mathscr{W}$ is a collection of open subsets of $E$ such that $h\left(\left(K \backslash K_{0}\right) \times I\right) \subset \mathscr{W}^{+}$. Then there exists a map $\varepsilon: \mathscr{W}^{+} \rightarrow(0, \infty)$ such that for every isotopy $\left(\psi_{t}\right)$ of $E$ with $\psi_{0}=\mathrm{id}, \psi_{t} \mid E \backslash \mathscr{W}^{+}=\mathrm{id}$ and $d\left(\psi_{t}(x), x\right)<\varepsilon(x)$ for all $x \in \mathscr{W}^{+}$and $t \in I$, the isotopy $\left(\psi_{t} \circ \phi_{t}\right)$ is limited by $\left\{\mathbf{s t}(h(k \times I), \mathbf{s t}(\mathscr{W})): k \in K \backslash K_{0}\right\}$.

Proof. Choose $\varepsilon: \mathscr{W}^{+} \rightarrow(0, \infty)$ with the property that whenever $h \in H(E)$, $h \mid E \backslash \mathscr{W}^{+}=$id and $d(h(x), x)<\varepsilon(x), x \in \mathscr{W}^{+}$, then $h$ is limited by $\mathscr{W}$; i.e., for each $x$ either $\{x, h(x)\}$ is contained in a member of $\mathscr{W}$ or it is one point. Now from $d\left(\psi_{t}(x), x\right)<\varepsilon(x)$ for some $t \in I$ it follows that $\left\{\psi_{t}\left(\phi_{t}(x)\right), \phi_{t}(x)\right\} \subset W, W \in \mathscr{W}$. Since $\phi(x \times I) \subset \operatorname{st}(h(k \times I), \mathscr{W})$ for some $k \in K \backslash K_{0}$ we infer that $\left\{\psi_{t}\left(\phi_{t}(x)\right): t \in I\right\} \subset \operatorname{st}(h(k \times I)$, st $(\mathscr{W}))$.

Proof of Theorem 6.1. Using 6.2 we may assume that the homotopy $\left(h_{t}\right)$ satisfies $h\left(\left(K \backslash K_{0}\right) \times I\right) \cap K_{0}=\varnothing$. By 6.3 there is a collection $\mathscr{V}$ of open subsets of $E$ such that $h\left(\left(K \cap \mathscr{U}^{+}\right) \times I\right) \subset \mathscr{V}^{+}$and $\left\{\operatorname{st}(h(k \times I), \mathscr{V}): k \in K \backslash K_{0}\right\}$ refines $\mathscr{U}$. Pick $\mathscr{W}$ open cover of $\mathscr{V}^{+}$such that $\operatorname{st}(\mathscr{W})$ refines $\mathscr{V}$. A required isotopy can be found as $\bar{h}_{t}=\psi_{t} \circ \phi_{t}$ with $\left(\phi_{t}\right)$ and $\left(\psi_{t}\right)$ satisfying the assumptions of 6.7. Consequently $\bar{h}=\left(\bar{h}_{t}\right)$ will be limited by $\mathscr{U}$. The isotopies $\left(\phi_{t}\right)$ and $\left(\psi_{t}\right)$ will be chosen so that $\phi_{1}=h_{0}$ and

$$
\psi_{1}=\lim \left(g_{0}^{-1} \circ \cdots \circ g_{n}^{-1} \circ g_{n+1}^{-1} \circ h_{n} \circ \cdots \circ h_{1}\right),
$$


where $h_{0}, h_{1}, \ldots$ and $g_{0}, g_{1}, \ldots$ are those of the proof of 1.3. The isotopy $\left(\phi_{t}\right)$ is that of Proposition 5.4 applied to the quadruple $\left(\left(K, K_{0}\right), U=\mathscr{W}^{+}, \varepsilon=\right.$ $\alpha, \mathscr{W})$, where $\alpha$ is that of 6.6 applied to the triple $\left(\left(h_{1}(K), h_{1}\left(K_{0}\right)\right), U=\mathscr{W}^{+}\right.$, $\varepsilon(x) / 4)$. Notice that $U$ contains the closure of $\left(h_{1}(K) \backslash h_{1}\left(K_{0}\right)\right)$. Using 6.6 we proceed by the inductive construction of 1.3 to get isotopies $g^{0}, g^{1}, \ldots$ and $h^{1}, h^{2}, \ldots$ of $E$. Finally we let $\phi_{t}=\lim \left(\left(g_{t}^{n+1} \circ \cdots \circ g_{t}^{0}\right)^{-1} \circ h_{t}^{n} \circ \cdots \circ h_{t}^{1}\right)$. The control functions $\varepsilon_{n}(x)$ are taken so small to get $d\left(\psi_{t}(x)\right)<\varepsilon(x)$ for some $x \in \mathscr{W}^{+}$. Since the convergence in the proof of 1.3 is uniform, the maps $(t, x) \rightarrow \psi_{t}(x)$ and $(t, x) \rightarrow \psi_{t}^{-1}(x)$ are continuous. Note that $\psi_{t}^{-1}(x)=$ $\lim \left(h_{t}^{n} \circ \cdots \circ h_{t}^{1}\right)^{-1} \circ g_{t}^{n+1} \circ \cdots \circ g_{t}^{0}(x)$. Hence the proof of 6.1 is completed.

Now we present another result concerning the controlled isotopy extension. We drop the assumption that $\mathscr{U}^{+}$contains the closure of $K \backslash \operatorname{Fix}(h)$.

6.8. Theorem. Suppose that a homotopy $\left(h_{t}\right)$ satisfies the hypothesis of 6.1. Then there exists an isotopy $\left(\bar{h}_{t}\right)$ limited by $\mathbf{s t}(\mathscr{U})$ and fulfilling the assertion of 6.1 .

We need the following lemma in which we slightly strengthen the fact that open subsets $U$ of $E$ have the discrete approximation property (see [11]), i.e., for every map $f: \bigoplus_{n=1}^{\infty} K_{n} \rightarrow U$ of disjoint union of compacta $\left(K_{n}\right)$ and for each open cover $\mathscr{W}$ of $U$ there exists a map $g: \bigoplus_{n=1}^{\infty} K_{n} \rightarrow U$ such that the collection $\left\{g\left(K_{n}\right)\right\}$ is discrete in $U$ and the family $\{f(x), g(x)\}, x \in$ $\bigoplus_{n=1}^{\infty} K_{n}$, refines $\mathscr{W}$.

6.9. Lemma. Let $V$ be an open subset of $E$ with $U \subseteq V$. Assume that the closure of $\operatorname{im}(f)$ in $V$ is contained in $U$ and that $\left\{f\left(L_{n}\right)\right\}$ is discrete in $V$, where $L_{n}$ is a closed subset of $K_{n}, n=1,2, \ldots$. Then there exists $\bar{f}: \bigoplus_{n=1}^{\infty} K_{n} \rightarrow U$ such that $\left\{\bar{f}\left(K_{n}\right)\right\}$ is discrete in $V, \bar{f}\left|L_{n}=f\right| L_{n}, \quad n=$ $1,2, \ldots$, and $\{f(x), \bar{f}(x)\}, x=\bigoplus_{n=1}^{\infty} K_{n}$, refines $\mathscr{W}$.

Proof. Let $\mathscr{U}^{\prime}=\mathscr{U} \cup(V \backslash \overline{\operatorname{im}(f)})$. There exists $g: \bigoplus_{n=1}^{\infty} K_{n} \rightarrow V$ such that $\left\{\left(g\left(K_{n}\right)\right\}\right.$ is discrete in $V$ and $d(f(x), g(x))<\varepsilon(f(x))$, where $\varepsilon: V \rightarrow(0, \infty)$ is a map such that $d(f(x), \phi(x))<\varepsilon(f(x))$ implies $\{f(x), \phi(x)\}$ refines $\mathscr{U}$, see [11]. Since $\left\{f\left(L_{n}\right)\right\}$ is discrete in $V$ we may assume that $\varepsilon(y)<1 / n$ for all $y \in f\left(L_{n}\right)$. Consider an open set $G_{n}=\left\{x \in K_{n}: d(f(x), g(x))<1 / n\right.$ and $\left.\operatorname{dist}\left(f(x), f\left(L_{n}\right)\right)<1 / n\right\}$. We see that $L_{n} \subset G_{n}$, so there exists a map $\lambda: \bigoplus_{n=1}^{\infty} K_{n} \rightarrow[0,1]$ with $\lambda \mid L_{n}=1$ and $\lambda \mid K_{n} \backslash G_{n}=0, n=1,2, \ldots$ Write $\bar{f}(x)=\lambda(x) f(x)+(1-\lambda(x)) g(x)$ for $x \in \bigoplus_{n=1}^{\infty} K_{n}$. Then

$$
d(f(x), \bar{f}(x))=d((1-\lambda(x))(f(x)-g(x)), 0) \leq d(f(x), g(x))<\varepsilon(f(x)) .
$$

From this it follows that $\{f(x), \bar{f}(x)\}$ refines $\mathscr{U}$ and $\operatorname{im}(\bar{f}) \subseteq U$. Let $\left\{\bar{f}\left(x_{k}\right)\right\}$, $x_{k} \in K_{n_{k}}$ and $n_{1}<n_{2}<\cdots$, be a sequence convergent in $V$. Since $\bar{f} \mid K_{n} \backslash G_{n}=$ $g \mid K_{n} \backslash G_{n}$ we may assume that $x_{k} \in G_{n_{k}}$. The inequality $d(f(x), \bar{f}(x)) \leq$ $d(f(x), g(x))$ yields the convergence of $\left\{f\left(x_{k}\right)\right\}$. Since $\operatorname{dist}\left(f\left(x_{k}\right), f\left(L_{n_{k}}\right)\right)<$ $1 / n_{k}$ the above inequality contradicts the fact that $\left\{f\left(L_{n}\right)\right\}$ was discrete. 
Proof of Theorem 6.8. First, according to 6.2, we replace the original homotopy by a homotopy $h$ such that $h\left(\left(K \backslash K_{0}\right) \times I\right) \cap K_{0}=\varnothing$, where $K_{0}=\operatorname{Fix}(h)$. Pick a sequence $\left\{U_{n}\right\}_{n=1}^{\infty}$ of open sets of $K$ such that $K=U_{0} \supset \bar{U}_{1} \supset U_{1} \supset \bar{U}_{2} \supset$ $U_{2} \supset \cdots$ and $\bigcap_{n=1}^{\infty} U_{n}=K_{0}$. Write $A_{i}=\bar{U}_{2 i-2} \backslash U_{2 i-1}$ and $B_{i}=\bar{U}_{2 i-1} \backslash U_{2 i}$, $i=1,2, \ldots$ We are going to construct a homotopy $h^{\prime}: K \times I \rightarrow E$ satisfying the following conditions: $h^{\prime}|K \times\{0,1\}=h| K \times\{0,1\}$, both collections $\left\{h^{\prime}\left(A_{i} \times I\right)\right\}$ and $\left\{h^{\prime}\left(B_{i} \times I\right)\right\}$ are discrete in $E \backslash K_{0}$ and $h^{\prime}$ is limited by $\mathscr{U}$. Let $\mathscr{V}$ be a collection of open sets in $E$ such that $\mathscr{V}^{+}$contains $h\left(\left(K \backslash K_{0}\right) \times I\right)$ and $\left\{\mathbf{s t}(h(k \times I), \mathscr{V}): k \in K \backslash K_{0}\right\}$ refines $\mathscr{U}$. There exists an open cover $\mathscr{W}$ such that $\mathrm{st}^{2}(\mathscr{W})$ refines $\mathscr{V}$. We apply 6.9 and approximate $\left\{h \mid A_{i} \times I\right\}$ by $\left\{g_{1} \mid A_{i} \times I\right\}$ so that $h=g_{1}$ on $A_{i} \times\{0,1\}$, the collection $\left\{g_{1}\left(A_{i} \times I\right)\right\}$ is discrete in $E \backslash K_{0}$ and $\left\{h(x), g_{1}(x)\right\}, x \in \bigcup A_{i} \times I$, refines $\mathscr{W}$. Note that the closure of $h\left(\left(K \backslash K_{0}\right) \times I\right)$ in $E \backslash K_{0}$ is contained in $\mathscr{W}^{+}$. Next $\left\{h \mid B_{i} \times I\right\}$ can be approximated by $\left\{g_{2} \mid B_{i} \times I\right\}$ so that $g_{2}=h$ on $B_{i} \times\{0,1\}$ and $g_{2}=g_{1}$ on $\left(\partial B_{i}\right) \times I$, where $\partial B_{i}$ is the boundary of $B_{i}$ in $K,\left\{g_{2}\left(B_{i} \times I\right)\right\}$ is discrete in $E \backslash K_{0}$ and $\left\{h(x), g_{2}(x)\right\}, x \in \bigcup B_{i} \times I$, refines $\mathrm{st}^{2}(\mathscr{W})$. To make this possible first we find a map $f:\left(K \backslash K_{0}\right) \times I \rightarrow E$ so that $f=g_{1}$ on $\bigcup\left(A_{i} \times I\right) \cup\left(K \backslash K_{0}\right) \times\{0,1\}$ and $\{h(x), f(x)\}, x \in\left(K \backslash K_{0}\right) \times I$, refines st $(\mathscr{W})$, and then we apply 6.9 to the map $f \mid \cup B_{i} \times I$, we see that $h^{\prime} \mid\left(K \backslash K_{0}\right) \times I$ given by $h^{\prime}\left|A_{i} \times I=g_{1}\right| A_{i} \times I$ and $h^{\prime}\left|B_{i} \times I=g_{2}\right| B_{i} \times I$ has the required properties. We may choose $g_{1}$ and $g_{2}$ in order that $h^{\prime}$ can be continuously extended to $K_{0} \times I$ by letting $h^{\prime}(x, t)=x$ for $(x, t) \in K_{0} \times I$. Further on use $h$ to denote the homotopy $h^{\prime}$. We enlarge the elements of $\left\{h\left(A_{i} \times I\right)\right\}$ and $\left\{h\left(B_{i} \times I\right)\right\}$ in order that the collections $\left\{B\left(h\left(A_{i} \times I\right), \delta_{i}\right)\right\}$ and $\left\{B\left(h\left(B_{i} \times I\right), \delta_{i}\right)\right\}$ remain discrete in $E \backslash K_{0}$; here $\delta_{i}>0$. For each $i$, let $\lambda_{i}: K \rightarrow I$ be a map satisfying $\lambda_{i} \mid A_{i}=1$ and $h\left(x, \lambda_{i}(x)\right) \in B\left(h\left(A_{i} \times I\right), \delta_{i} / 2\right)$ provided that $\lambda_{i}(x) \neq 0$. Each map $\lambda_{i}$ determines a homotopy $f^{i}: K \times I \rightarrow E$ by $f^{i}(x, t)=h\left(x, \lambda_{i}(x) \cdot t\right)$. Clearly, we have $f_{0}^{i}=\mathrm{id}, f_{1}^{i}(x, t)=h\left(x, \lambda_{i}(x)\right)$, $f^{i}=h$ on $A_{i} \times I$, the family $\left\{f^{i}\left(\left(K \backslash \operatorname{Fix}\left(f^{i}\right)\right) \times I\right)\right\}$ is discrete in $E \backslash K_{0}$, and $f^{i}$ is limited by $\mathscr{U}$. It is easily seen that the family $\left(f^{i}\right)$ determines a homotopy $f: K \times I \rightarrow E$ equal to $f^{i}$ on $\left(K \backslash \operatorname{Fix}\left(f^{i}\right)\right) \times I$ and the identity elsewhere. Next we approximate $f_{1}: K \rightarrow E$ by an embedding $u: K \rightarrow E$ such that $u\left|A_{i}=f_{1}^{i}\right| A_{i}=h_{i} \mid A_{i}$ and $u \mid \operatorname{Fix}(f)=\mathrm{id}$. We can do it applying 4.3 to each $f^{i}$. Consider the segment homotopy $u_{t}(x)=t u(x)+(1-t) f_{1}(x)$ connecting $f_{1}$ with the embedding $u_{1}=u$. A homotopy $g: K \times I \rightarrow E$ which is the composition of $f$ and $\left(u_{t}\right)$ joins the identity with $u$. Consider the homotopy $g^{i}: K \times I \rightarrow E$ equal to $\left(u_{t}\right)$ on $K \backslash \operatorname{Fix}\left(F^{i}\right)$ and $u_{i}(x)=x$ elsewhere. If $u$ is close enough to $f_{1}$ then $g^{i}\left(\left(K \backslash \operatorname{Fix}\left(g^{i}\right)\right) \times I\right) \subset B\left(h\left(A_{i} \times I\right), \delta_{i}\right)$ and therefore the collection $\left\{g^{i}\left(\left(K \backslash \operatorname{Fix}\left(g^{i}\right)\right) \times I\right)\right\}$ is discrete in $E \backslash K_{0}$. Moreover if $u$ is sufficiently close to $f_{1}$ then $g$, and consequently $g^{i}$, will be limited by $\mathscr{U}$. The next step is to connect homotopically $g_{1}$ with $h_{1}$. Observe that 
the homotopy $\left(u_{1-t}\right)$ joins $u$ with $f_{1}(x)=h(x, \lambda(x))$ while the homotopy $(x, t) \rightarrow h(x,(1-t) \lambda(x)+t)$ joins $f_{1}$ with $h_{1}$. Thus their composition $\left(p_{t}^{\prime}\right)$ joins $u$ with $h_{1}$. Consider $C_{i}=u\left(B_{i}\right)$ and let $p_{t}^{i}(y)=p_{t}^{\prime}\left(u^{-1}(y)\right)$ for $y \in C_{i}$ and $i=1,2, \ldots$. Then $\left(p_{t}^{i}\right)$ joins $\operatorname{id}_{C_{i}}$ with $h_{1} \circ u^{-1} \mid C_{i}$. Since $p_{t}^{i}(y)=y$ for $y \in \partial C_{i}$, the boundary $\partial C_{i}$ is taken in $u(K)$, the homotopy $\left(p_{t}^{i}\right)$ can be extended to the whole $u(K)$ by letting $p_{t}^{i}(y)=y$ off $C_{i}$. If the map $u$ is sufficiently close to $f_{1}$ then $p^{i}\left(\left(u(K) \backslash \operatorname{Fix}\left(p^{i}\right)\right) \times I\right) \subset B\left(h\left(B_{i} \times I\right), \delta_{i}\right)$ and thus the collection $\left\{p^{i}\left(\left(u(K) \backslash \operatorname{Fix}\left(p^{i}\right)\right) \times I\right)\right\}$ will be discrete in $E \backslash K_{0}$, moreover $p^{i}$ will be limited by $\mathscr{U}$. Now to each homotopy $g^{i}$ and $p^{i}$ we apply Theorem 6.1. There are isotopies $G^{i}$ and $P^{i}$ of $E$ such that $G_{0}^{i}=\mathrm{id}, P_{0}^{i}=\mathrm{id}$, $G_{1}^{i}=u$ on $K \backslash \operatorname{Fix}\left(f^{i}\right), P_{1}^{i}=p_{1}^{i}$ and $\operatorname{Fix}\left(P^{i}\right)=\operatorname{Fix}\left(\left(p_{t}^{i}\right)\right)$. The isotopies $G^{i}$ and $P^{i}$ are limited by a collection $\mathscr{V}$ finer than $\mathscr{U}$ in order that the families $\left\{E \backslash \operatorname{Fix}\left(G^{i}\right)\right\}$ and $\left\{E \backslash \operatorname{Fix}\left(P^{i}\right)\right\}$ are discrete in $E \backslash K_{0}$ and the sequences $\sup \left\{\operatorname{dist}\left(y, E \backslash \operatorname{Fix}\left(g^{i}\right)\right): y \notin \operatorname{Fix}\left(G^{i}\right)\right\}$ and $\sup \left\{\operatorname{dist}\left(y, E \backslash \operatorname{Fix}\left(p^{i}\right)\right): y \notin\right.$ Fix $\left.\left(P^{i}\right)\right\}$ tend to zero. We see that the isotopies $\left(G^{i}\right)$ and $\left(P^{i}\right)$ determine isotopies $G$ and $P$ on $E \backslash K_{0}$ given by $G(x)=G^{i}(x)$ for $x \in E \backslash \operatorname{Fix}\left(G^{i}\right)$ and $P(x)=P^{i}(x)$ for $x \in E \backslash \operatorname{Fix}\left(P^{i}\right)$. It can be easily checked that, letting $G(x, t)=P(x, t)=x$ for $(x, t) \in K_{0} \times I, G$ and $P$ extend to isotopies of the whole $E$. Finally the composition of $G$ and $P$ defines a required isotopy $\bar{h}$ which is limited by $\mathrm{st}(\mathscr{U})$.

6.10. Remark. Let $K^{\prime}=K \times I \sim$, where $\left(x_{1}, t\right) \sim\left(x_{2}, s\right)$ iff $x_{1}=x_{2} \in \operatorname{Fix}(h)$. Furthermore, let $h^{\prime}: K^{\prime} \rightarrow E$ be defined by $h^{\prime}([(x, t)])=h_{t}(x)$. (Note that $K^{\prime}$ is a compact metrizable space.) If we assume that $K^{\prime}$ admits an embedding into $E$ then in the assertion of 6.8 we may additionally require that the isotopy $\bar{h}$ is limited by $\mathscr{U}$. In this case we may replace the original homotopy by a homotopy $h$ such that $h \mid\left(K \backslash K_{0}\right) \times I$ is an embedding. Then use the fact that for every $\varepsilon>0$, there exists $\delta_{n}>0$ such that $\sup \left\{d\left(h_{s}(x), h_{s}(y)\right): s \in I\right\}<\varepsilon$ provided $d\left(h_{t}(x), h_{u}(y)\right)<\delta_{n}$ for some $t, u \in I$ and every $x, y \in A_{n} \cup B_{n} \cup A_{n-1}$.

Here is our main result concerning controlled isotopy extension for local compacta.

6.11. Theorem. Let $E$ be an infinite-dimensional l.c.m.l.s. and let $A$ be its closed locally compact subset. Let $h=\left(h_{t}\right)$ be a homotopy of $A$ into $E$ such that $h_{0}=$ id and $h_{1}: A \rightarrow h_{1}(A)$ is a closed embedding. Assume that $h$ is limited by a collection $\mathscr{U}$ of open subsets of $E$. If the closure of $h(A \backslash \operatorname{Fix}(h))$ is contained in $\mathscr{U}^{+}$then there exists an isotopy $\left(\bar{h}_{t}\right)$ of E limited by $\operatorname{st}(\mathscr{U})$ with $\bar{h}_{0}=\mathrm{id}, \bar{h}_{1} \mid A=h_{1}$ and $\bar{h}_{t} \mid \operatorname{Fix}(h)=\mathrm{id}, t \in I$.

Proof. We repeat the argument of 6.8. We replace the original homotopy by a homotopy $h$ with $h\left(\left(A \backslash A_{0}\right) \times I\right) \cap A_{0}=\varnothing$, where $A_{0}=\operatorname{Fix}(h)$. Then we pick a sequence $\left\{U_{n}\right\}_{n=0}^{\infty}$ of open subsets of $A$ such that each $A \backslash U_{n}$ is compact, $A=U_{0} \supset \bar{U}_{1} \supset U_{1} \supset \bar{U}_{2} \supset U_{2} \supset \cdots$ and $\bigcap_{n=1}^{\infty} U_{n}=\varnothing$. Define the sets $A_{i}$ 
and $B_{i}$ as in the proof of 6.8. The condition that $\operatorname{cl}\left(h\left(\left(A \backslash A_{0}\right) \times I\right)\right) \subset \mathscr{U}^{+}$ allows us 10 modify Lemma 6.9 so that we are able to construct a homotopy $h^{\prime}: A \times I \rightarrow E$ which is limited by $\mathscr{U}$ and such that $h^{\prime}|A \times\{0,1\}=h| A \times\{0,1\}$ and the collections $\left\{h^{\prime}\left(A_{i} \times I\right)\right\}$ and $\left\{h^{\prime}\left(B_{i} \times I\right)\right\}$ are discrete in the whole space $E$. Further on we proceed as in the proof of 6.8. While constructing $G^{i}$ and $P^{i}$ we additionally require that $G_{t}^{i}\left|A_{0}=P_{t}^{i}\right| A_{0}=\mathrm{id}, t \in I$ and $i=1,2, \ldots$. This time the families $\left\{E \backslash \operatorname{Fix}\left(G^{i}\right)\right\}$ and $\left\{E \backslash \operatorname{Fix}\left(P^{i}\right)\right\}$ are discrete in the whole $E$. Hence the step of extending $G$ and $P$ to $A_{0}$ can be omitted. An isotopy $\bar{h}$ is the composition of $G$ and $P$.

An immediate consequence of Theorem 6.11 is the following generalization of 2.12 .

6.12. Corollary. Every infinite-dimensional locally convex metric linear space $E$ has the homeomorphism extension property for local compacta, i.e., every homeomorphism between closed local compacta of $E$ extends to a homeomorphism of $E$.

Let $E$ be an infinite-dimensional l.c.m.l.s. containing $E$ as a dense linear subspace. All the isotopies $\left(\bar{h}_{t}\right)$ appearing in 6.4, 6.6 and in the proofs of 6.1, 6.8, 6.11 and 6.12 can be taken to satisfy $\bar{h}_{t} \in H(E \mid E), t \in I$. This makes it possible to claim

6.13. Remark. In Theorems $6.1,6.8,6.11$ and 6.12 we may additionally require that $\bar{h}_{t} \in H(E \mid E), t \in I$, and $\bar{h} \in H(E \mid E)$, respectively.

\section{REFERENCES}

1. R. D. Anderson, On sigma-compact subsets of infinite-dimensional spaces, unpublished manuscript.

2. R. D. Anderson and J. D. McCharen, On extending homeomorphisms to Fréchet manifolds, Proc. Amer. Math. Soc. 25 (1970), 283-289.

3. C. Bessaga and A. Pelczyński, The estimated extension theorem homogeneous collections and skeletons and their applications to the topological classification of linear metric spaces and convex sets, Fund. Math. 69 (1970), 153-190.

4. $\ldots$, Selected topics in infinite-dimensional topology, Polish Scientific Publishers, Warsaw, 1975.

5. J. Bryant, On embeddings of compacta in euclidean space, Proc. Amer. Math. Soc. 23 (1969), 46-51.

6. D. Curtis, T. Dobrowolski and J. Mogilski, Some applications of the topological characterization of the sigma-compact spaces $l_{2}^{f}$ and $\Sigma$, Trans. Amer. Math. Soc. 284 (1984), 837-846.

7. T. Dobrowolski, Smooth and R-analytic negligibility of subsets and extension of homeomorphisms in Banach spaces, Studia Math. 65 (1979), 115-139.

8. __ On extending mappings into nonlocally convex linear metric spaces, Proc. Amer. Math. Soc. 93 (1985), 555-560.

9. __ The compact Z-set property in convex sets, Topology Appl. 23 (1986), 163-172.

10. T. Dobrowolski and J. Mogilski, Sigma-compact locally convex metric linear spaces universal for compacta are homeomorphic, Proc. Amer. Math. Soc. 78 (1982), 653-658. 
11. T. Dobrowolski and H. Toruńczyk, Separable complete ANRs admitting group structures are Hilbert manifolds, Topology Appl. 12 (1981), 229-235.

12. H. Gluck, Embeddings in trivial range, Ann. of Math. (2) 81 (1965), 195-210.

13. T. Homma, On the embedding of compacta in manifolds, Yokohama Math. J. 10 (1962), 5-10.

14. V. L. Klee, Some topological properties of convex sets, Trans. Amer. Math. Soc. 78 (1955), 30-45.

15. E. Michael, Convex structures and continuous selections, Canad. J. Math. 11 (1954), 556-575.

16. M. A. Štanko, The embedding of compacta in euclidean space, Mat. Sb. 12 (1970), 234-254.

17. H. Torunczyk, Skeletonized sets in complete metric spaces and homeomorphisms of the Hilbert cube, Bull. Acad. Polon. Sci. Ser. Sci. Math. Astronom. Phys. 18 (1970), 119-126.

18. __ Smooth partitions of unity in some non-separable Banach spaces, Studia Math. 46 (1973), 43-51.

19. Concerning locally homotopy negligible sets and characterizations of $l_{2}$-manifolds, Fund. Math. 101 (1978), 93-110.

20. __ Characterizing Hilbert space topology, Fund. Math. 111 (1981), 247-262.

Institute of Mathematics, Warsaw University, PKiN IX P., 00-901 Warsaw, Poland 